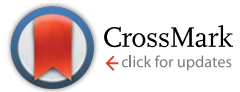

Cite this: Soft Matter, 2015, 11, 3251

\section{The rheology of aqueous solutions of ethyl hydroxy-ethyl cellulose (EHEC) and its hydrophobically modified analogue (hmEHEC): extensional flow response in capillary break-up, jetting (ROJER) and in a cross-slot extensional rheometer $\dagger$}

\author{
Vivek Sharma, ${ }^{\text {*ab }}$ Simon J. Haward, ${ }^{\text {bc }}$ James Serdy, ${ }^{\text {d }}$ Bavand Keshavarz, ${ }^{\text {b }}$ \\ Asa Soderlund, ${ }^{e}$ Phil Threlfall-Holmes ${ }^{f}$ and Gareth H. McKinley ${ }^{b}$
}

Cellulose derivatives containing associating hydrophobic groups along their hydrophilic backbone are used as rheology modifiers in the formulation of water-based spray paints, medicinal sprays, cosmetics and printable inks. Jetting and spraying applications of these materials involve progressive thinning and break-up of a fluid column or sheet into drops. Strong extensional kinematics develop in the thinning fluid neck. In viscous Newtonian fluids, inertial and viscous stresses oppose the surface tension-driven instability. In aqueous solutions of polymers such as Ethyl Hydroxy-Ethyl Cellulose (EHEC), chain elongation provides additional elastic stresses that can delay the capillary-driven pinch-off, influencing the sprayability or jettability of the complex fluid. In this study, we quantify the transient response of thinning filaments of cellulose ether solutions to extensional flows in a Capillary Break-up Extensional Rheometer (CaBER) and in a forced jet undergoing break-up using Rayleigh Ohnesorge Jetting Extensional Rheometry (ROJER). We also characterize the steady state molecular deformations using measurements of the flow-induced birefringence and excess pressure drop in an extensional stagnation point flow using a Cross-Slot Extensional Rheometer (CSER). We show that under the high extension rates encountered in jetting and spraying, the semi-dilute solutions of hydrophobically modified ethyl hydroxy-ethyl cellulose (hmEHEC) exhibit extensional thinning, while the unmodified bare chains of EHEC display an increase in extensional viscosity, up to a plateau value. For both EHEC and hmEHEC dispersions, the low extensibility of the cellulose derivatives limits the Trouton ratio observed at the highest extension rates attained (close to $10^{5} \mathrm{~s}^{-1}$ ) to around 10-20. The reduction in extensional viscosity with increasing extension rate for the hydrophobically modified cellulose ether is primarily caused by the disruption of a transient elastic network that is initially formed by intermolecular association of hydrophobic stickers. This extensional thinning behavior, in conjunction with the low extensibility of the hydrophobically modified cellulose ether additives, makes these rheology modifiers ideal for controlling the extensional rheology in formulations that require jetting or spraying, with minimal residual stringiness or stranding.
Received 28th July 2014

Accepted 26th January 2015

DOI: $10.1039 / \mathrm{c} 4 \mathrm{sm} 01661 \mathrm{k}$

www.rsc.org/softmatter

\section{Introduction}

E-mail: viveks@uic.edu

${ }^{b}$ Hatsopoulos Microfluids Laboratory, Department of Mechanical Engineering, Massachusetts Institute of Technology, Cambridge, MA 02139-4307, USA

'Okinawa Institute of Science and Technology Graduate University, 1919-1 Tancha, Onna-son, Kunigami-gun, Okinawa 904-0495, Japan

${ }^{d}$ Laboratory of Manufacturing and Productivity, Department of Mechanical Engineering, Massachusetts Institute of Technology, Cambridge, MA 02139-4307, USA ${ }^{e}$ AkzoNobel Functional Chemicals, Hamnvägen 2, Stenungsund 444 85, Sweden ${ }^{f}$ AkzoNobel Research, Development and Innovation, Stoneygate Lane, Felling, Gateshead, NE10 OJY, UK

$\dagger$ Electronic supplementary information (ESI) available. See DOI: $10.1039 / \mathrm{c} 4 \mathrm{sm} 01661 \mathrm{k}$
Multicomponent complex fluids containing long polymer molecules are found to provide a much larger resistance to extensional flow than expected on the basis of their shear viscosity. For a Newtonian fluid, the extensional viscosity is a factor of three times larger than the shear viscosity (as was first shown by Trouton ${ }^{1}$ ); however it can be several orders of magnitude higher for polymeric fluids. ${ }^{1-3}$ A stretching or extensional deformation is established when streamwise velocity gradients are present in a flow. Such extensional 
components arise in virtually all relevant processing flows, ${ }^{2-4}$ including capillary contraction/expansion flows, flows through bifurcations (e.g. 'T' or 'Y' junctions), flow around obstacles and near stagnation points, ${ }^{5-7}$ as well as in common industrial processes such as spraying, jet break-up, drop formation and filament stretching..$^{8-11}$ Extensional viscosity enhancement can dramatically influence the flow characteristics of fluids in these processes. ${ }^{\text {2-1-11 }}$ Designing fluids with appropriate characteristics (which are commonly expressed in heuristic terms such as sprayability, spinnability, printability and jettability) requires systematic control over the response of a complex fluid to both shear and extensional deformations. Polysaccharides or carbohydrate polymers ${ }^{\mathbf{1 2 - 1 4}}$ are among the most common constituents of industrial and natural complex fluids and thus contribute to the measurable flow resistance in many extensional deformations, influencing spraying and coating of paints, ${ }^{15}$ inkjet printing, filament spinning, ${ }^{16}$ porous media flow, ${ }^{17}$ enhanced oil recovery, ${ }^{18}$ and turbulent drag reduction as well as in natural systems such as synovial fluid, mucus and deadly viscoelastic fluids found in carnivorous plants. ${ }^{19,20}$

In this work we contrast the shear and extensional rheology of model aqueous solutions of cellulose ether, EHEC (Ethyl Hydroxy-Ethyl Cellulose), and its hydrophobically modified analogue, hmEHEC. The hmEHEC chains have hydrophobic stickers distributed along the backbone. In aqueous dispersion, the hydrophobic stickers associate together as temporary junctions that break and reform continuously under the action of thermal fluctuations and in response to applied deformations. ${ }^{21-25}$ Since these cellulose ethers are used as rheology modifiers in multi-component fluids, we probe their response over the wide range of shear and extensional rates that characterize typical process flows. In a companion study, we discuss the shear-rate-dependent response of EHEC and hmEHEC dispersions as manifested by the shear viscosity and first normal stress difference measurements for different polymer concentrations. Interchain hydrophobic associations between the multisticker hmEHEC polymers lead to the formation of transient physical gels that provide enhancement to the low shear-rate viscosity when compared with dispersions of bare EHEC chains. At high shear rates, the gel-like microstructure breaks down and the high shear rate viscosity of the sticky, hydrophobically modified chains becomes quite similar to that of the bare polymer. We infer that the enhancement in viscosity due to stickers at low shear rates provides the requisite high viscosity for controlling sagging and slumping behavior of paints, ${ }^{26}$ while the high degree of shear thinning associated with the break-down of the microstructure leads to a much lower shear viscosity at the high shear rates most relevant to coating applications. We also characterize the linear viscoelasticity of the physical networks formed by the sticky hmEHEC polymers using a Fractional Maxwell Model (FMM). Additionally we contrasted the measured concentration-dependent response of the linear viscoelasticity of these sticky polymer networks with the theoretical predictions of Rubinstein and Semenov. ${ }^{27,28}$ In the present paper, we focus exclusively on a comparison between the extensional behavior of aqueous dispersions of cellulose ether, EHEC and the corresponding multisticker associative polymer, hmEHEC. We probe the influence of transient extensional rheology at moderate extensional rates during capillary thinning break-up and at higher deformation rates using the free-surface flow during jetting. We also measure extensional contributions to the bulk pressure drop and the corresponding molecular deformation in a steady extensional flow field generated within a cross-slot flow extensional rheometer. We specifically consider the rheological properties from the perspective of design and application of water-based paints, to put into context the role of associative polymers as rheology modifiers in industrial applications.

The application of paint to surfaces commonly involves the use of either roller-coating devices or spraying equipment. ${ }^{29,30}$ Both of these application processes involve the formation of elongated fluid filaments that spontaneously break into droplets, due to a capillary-driven instability that seeks to minimize the total surface area of the fluid sample. This surface-tensiondriven instability is manifested as sinusoidal perturbations to the initial cylindrical shape of the fluid column, and this creates a uniaxial extensional flow field within the necking filament, as the enhanced local capillary pressure squeezes fluid out of the narrow neck..$^{\mathbf{8 , 9}, 31}$ The process of capillary thinning eventually leading to pinch-off is opposed by the presence of viscous and inertial stresses. ${ }^{32-34}$ In polymeric complex fluids, microstructural changes and the associated increase in the total viscous drag on the elongating polymer chains provide extra elastic stresses that can substantially delay the capillary-driven pinchoff as well as modify the drop size and the distribution of drop sizes. ${ }^{\mathbf{8 1 3}, \mathbf{3 5 - 4 4}}$ In spite of the widespread use of polysaccharides or cellulose derivatives (with and without associative stickers) as rheology modifiers in applications where the response to extensional flows determines their utility, their response to extensional deformation, especially in the context of jetting and spraying, remains a less well-studied problem. ${ }^{12}$ The lack of requisite characterization at high deformation rates for viscoelastic fluids is due to the difficulties and limitations inherent to the techniques commonly used for measuring the response to extensional flow fields, as we describe next.

Extension-free simple shear flows can be readily generated in cone-and-plate or Couette geometries on a torsional rheometer ${ }^{4}$ in order to measure the rate-dependent shear viscosity, $\eta(\dot{\gamma})$, as well as the first and second normal stress differences, $N_{1}(\dot{\gamma})$ and $N_{2}(\dot{\gamma})$. Likewise, it is desirable to measure the extensional viscosity, $\eta_{\mathrm{E}}(\dot{\varepsilon})$, of a complex fluid as the material response to a shear-free, purely extensional deformation where the imposed extensional rate, $\dot{\varepsilon}$, is controlled to be constant. However, the extensional response of complex fluids in any geometry or device exhibits a strong dependence on flow parameters ${ }^{9}$ including both the strain rate, $\dot{\varepsilon}$, and the total fluid strain, $\varepsilon$. Thus the measured extensional viscosity is a deformationhistory dependent material function, and the underlying flow kinematics in most techniques result in measurement of a transient extensional viscosity or $\eta_{\mathrm{E}}^{\dagger}(\dot{\varepsilon}, t) .{ }^{45}$ Fig. 1 summarizes various techniques ${ }^{2,4}$ that can be used for studying polymer solution extensional rheology. The typical range of zero shear viscosity and extensional rates accessed by each device are shown qualitatively. Though the comparison of different 


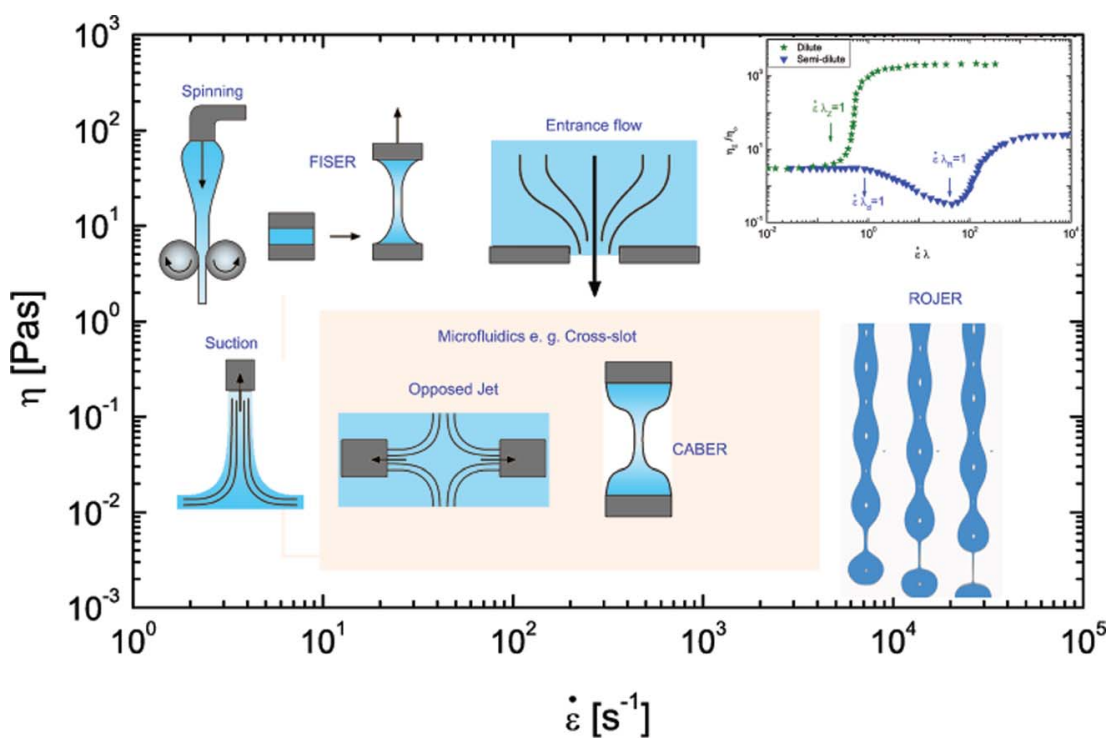

Fig. 1 Extensional rheometry of polymer solutions: typical techniques used for measuring the response of polymer solutions and their typical measurement range. The choice of measurement techniques is guided both by the magnitude of the zero shear viscosity (plotted on ordinate) and the range of extension rates accessible by the technique (on abscissa). Inset: typical rate-dependent extensional viscosity response displayed by dilute and semi-dilute solutions. Note that the ratio of extensional viscosity to zero shear viscosity plotted on the ordinate is commonly referred to as the Trouton ratio, while the abscissa corresponds to the Weissenberg number which quantifies the extensional flow strength by comparing the deformation rate with relaxation time.

measures of extensional viscosity characterized using different techniques can show a great disparity, ${ }^{45,46}$ understanding the dynamics of the response from a given method is extremely useful in designing complex fluids with the requisite processability, or alternatively in controlling the processing parameters for a given complex fluid. The use of experimental techniques with well-controlled flow fields and deformation histories is also important for developing constitutive models for complex fluids. There is also considerable growth in recent interest in designing suitable, polymeric complex fluids for additive manufacturing processes where relating the processability to the underlying fluid rheology will be necessary for realizing economics of scale and quality in 3-D printing, ink-jet printing-based biomaterials, print electronics and print photovoltaics. ${ }^{\text {10,47-49 }}$

Steady extension-dominated flows that enable control over both the local strain rate and the total accumulated strain can be realized by incorporating a stagnation point in the flow field. ${ }^{50-53}$ At such a singular point, the local fluid velocity approaches zero but the strain rate can be large. Hence macromolecules contained in the fluid elements that pass through the stagnation point become trapped in the elongational flow field for an extended time period and can accumulate significant strain provided the characteristic deformation rate exceeds $\dot{\varepsilon}>1 / \lambda$, where $\lambda$ is the longest relaxation time of the polymeric fluid. The relative magnitude of the relaxation rate $(1 / \lambda)$ and deformation rate $(\dot{\varepsilon})$ is characterized by the Weissenberg number, $\mathrm{Wi}=\lambda \dot{\varepsilon}$, and extensional flows with $\mathrm{Wi}>1$ are characterized as strong flows. Stagnation point flows can be generated using macroscopic techniques like four-roll mills and opposed jet or cross-slot devices, ${ }^{54}$ and the measurements can be extended to higher rates by using microfluidic devices ${ }^{50,55}$ such as T-junctions ${ }^{56,57}$ four-roll mill analogues ${ }^{58-61}$ and crossslots, ${ }^{6,53,62-68}$ where the small length scales and lower flow rates required to achieve high Weissenberg numbers result in reduced inertial contributions to the flow. By a suitable choice of geometry, such microfluidic devices allow measurements up to extension rates of at least $10^{4} \mathrm{~s}^{-1}$ and most of these techniques also allow the use of rheo-optical methods for visualizing both flow kinematics and the polymer orientation and stretch. ${ }^{50}$ In the present study, we use a Cross-Slot Extensional Rheometer (CSER) to measure both the change in birefringence and the extra pressure drop near a stagnation point to characterize the extensional deformation of the cellulose ether solutions.

Quantitative analysis of the capillary-thinning dynamics of complex fluids can also provide a measure of the transient extensional viscosity and chain unraveling timescale relevant for capillary-driven flows. ${ }^{9,40,68-75}$ Measurement of the capillarydriven self-thinning dynamics of a stretched liquid bridge is the underlying principle of the Capillary Break-up Extensional Rheometer (CaBER). ${ }^{9,42,76,77}$ In this device, the practical limit to the measurable response is set by the time required to stretch the liquid bridge $\mathrm{e}^{77}$ and more specialized bespoke instrumentation must be developed for low viscosity systems. ${ }^{73,74}$ We have recently developed a jetting-based rheometry technique, called the Rayleigh Ohnesorge Jetting Extensional Rheometer (ROJER) that provides access to the typically unchartered regime of very short relaxation time, low viscosity complex fluids, ${ }^{70,71}$ corresponding to the bottom right corner of Fig. 1. The rheometry technique is based on the understanding of the nonlinear fluid dynamics underlying the jetting process, as described in the text later, and is motivated by a method originally suggested by Schümmer and Tebel. ${ }^{78}$ In the present study, we examine the 
capillary break-up dynamics in both CaBER and ROJER devices in order to understand the extensional behavior of cellulose ethers in free-surface flow configurations that closely mimic the deformation history and stresses encountered in jetting and spraying applications.

The typical characteristics of extensional viscosity responses for dilute and semi-dilute polymer solutions are shown in the inset to Fig. 1. The enhanced resistance to extensional deformation is most pronounced and well-studied for dilute polymer solutions where a macromolecular conformational change from a random coil to a highly stretched state underlies the observed increase in resistance to elongational flow. Extensional viscosities up to $10^{2}$ to $10^{4}$ times larger than the zero shear viscosity have been reported for dilute solutions ${ }^{75}$ and the values increase with molecular weight, as indicated schematically by the inset (Fig. 1). In semi-dilute solutions, due to the overlap between different chains, the effective strain encountered by any individual coil is lower. Entangled semi-dilute solutions (blue triangles) thus typically show a regime of extensional thinning followed by extensional thickening at higher rates. ${ }^{79-83}$ However, depending on the entanglement density and chain rigidity, some dilute and semi-dilute solutions show an extensional thickening regime followed by extensional thinning at high rates (see open symbols). ${ }^{\mathbf{5 2 2 , 8 4 , 8 5}}$ In this paper, the measured response of polymer chains with self-associating or 'sticky' side groups (hmEHEC dispersions) is contrasted with the corresponding response of bare polymer chains (EHEC dispersions). The understanding of the response of multisticker polymer chains is a necessary step to arrive at a rational basis for choosing or designing optimal water-soluble rheology modifiers for jetting and spraying applications.

\section{Materials and methods}

\subsection{Materials}

In this study, we use a hydrophobically modified ethyl hydroxyethyl cellulose ether (hmEHEC) with the degree of substitution (DS) occupied by the ethyl group given by $\mathrm{DS}_{\mathrm{Et}} \approx 0.8$; the molar degree of substitution (MS) by ethylene oxide groups is $\mathrm{MS}_{\mathrm{EO}} \approx$ 2.5 , and the degree of substitution by the hydrophobic sticker distributed along the chain is $\mathrm{MS}_{\mathrm{C} 14} \approx 0.008 .^{15}$ The hmEHEC was provided in a purified form by Åsa Söderlund, AkzoNobel Cellulosic Specialties and has a degree of polymerization DP $\approx$ 800 , which corresponds to a molecular weight $M_{\mathrm{w}} \approx 240000$ Daltons. The molecular weight of the individual unsubstituted anhydrous glucose unit is $M_{0}=162$ Daltons. In addition to hmEHEC, which we often refer to as a 'sticky' polymer in the rest of this paper, we also use the corresponding unmodified or 'bare' polymer (EHEC), which has no hydrophobic stickers along the chain but has a similar molecular weight and degree of substitution. The shear rheology of both the polymers is described in detail in a companion paper.

\subsection{Cross-slot extensional rheometer (CSER)}

We studied the steady extensional flow of three representative EHEC and hmEHEC solutions in a microfluidic cross-slot device. Cross-slot devices consist of orthogonal, mutually bisecting channels with opposing inlets and outlets, which, by virtue of their symmetry, generate a stagnation point at the exact center of the flow domain. ${ }^{86}$ The strain rate $\left(\dot{\varepsilon}_{\mathrm{CS}}\right)$ is controlled by the volumetric flow rate through the device and we can perform measurements of flow-induced birefringence in the vicinity of the stagnation point together with the macroscopic pressure loss across the channel as the flow rate is progressively increased. An optical micrograph of the cross-slot geometry used in the study is shown in Fig. 2a. We define the $x$ and $y$ axes as shown in the figure, with the stagnation point (marked by the blue ' $x$ ' at the centre of the intersecting channels) taken as the coordinate origin and the $z$-axis normal to the plane of the page. The slots are precision machined with a width of $w=0.2 \mathrm{~mm}$, and a depth in the $z$-direction of $d=1050 \pm$ $10 \mu \mathrm{m}$, providing an aspect ratio $\alpha=d / w \approx 5$ and hence a quasi$2 \mathrm{D}$ flow. The length of the inlet/outlet channels is $l=1.2 \mathrm{~mm}$. In all the results presented herein the inflow and outflow directions are as marked in Fig. 2a.

The cross-slots are fabricated from stainless steel discs by the technique of wire electrical discharge machining (wire-EDM). This method provides highly parallel, non-tapering walls and a smooth surface finish; both of which are essential features for producing a stable, symmetric flow. Annealed soda glass viewing windows are glued to the front and rear surfaces of the stainless steel flow channel (see Fig. 2b), allowing optical access to the region near the stagnation point. The rear window first has four holes drilled through it ultrasonically, to allow flow into/out of each arm of the device. Finally, the stainless steel/glass sandwich assembly is glued onto a monolithic stainless steel back-plate, which provides connections to the external plumbing and flow loop, as shown in Fig. 2c. All bonds are made using silicone aquarium adhesive. The continuous flow through the cross-slot device is driven at a controlled rate using a precision syringe pump (Harvard PHD-Ultra). The flow from the syringe pump, labeled (1) in Fig. 2c, is split into two channels to provide the flow into the opposing inlets of cross-slot (3). The two outlets are connected together so they remain at equal pressure and do not generate an unbalanced flow. One of the inlet channels is fitted with a $35 \mathrm{kPa}$ gauge pressure transducer (GE Druck) (2) to measure the pressure drop across the flow cell. Effluent is ejected to a Petri dish (5) and discarded to waste.

The syringe pump delivers volume flow rates in the range $0.1 \mathrm{~mL} \min ^{-1} \leq Q \leq 10 \mathrm{~mL} \mathrm{~min}{ }^{-1}$. This provides nominal extension rates at the stagnation point of $40 \mathrm{~s}^{-1} \leq \dot{\varepsilon}_{\mathrm{CS}} \leq$ $4000 \mathrm{~s}^{-1}$, that are calculated according to the expression:

$$
\dot{\varepsilon}_{\mathrm{CS}}=\frac{Q}{w^{2} d}
$$

The Reynolds number for flow in the cross-slot device is calculated using $\operatorname{Re}=\rho U D_{\mathrm{h}} / \eta(\dot{\gamma})$, where $D_{\mathrm{h}}$ is the hydraulic diameter, $D_{\mathrm{h}}=2 w d /(w+d)$, and $\eta(\dot{\gamma})$ is the shear rate-dependent shear viscosity. Within the cross-slot, assuming an ideal planar extensional flow given by $\mathbf{u}=\left[\dot{\varepsilon}_{\mathrm{CS}} x,-\dot{\varepsilon}_{\mathrm{CS}} y, 0\right]^{T}$ the corresponding value of the characteristic shear rate is $\dot{\gamma}=\sqrt{\frac{1}{2} \mathrm{II}(\dot{\gamma})}=2 \dot{\varepsilon}_{\mathrm{CS}}$, 

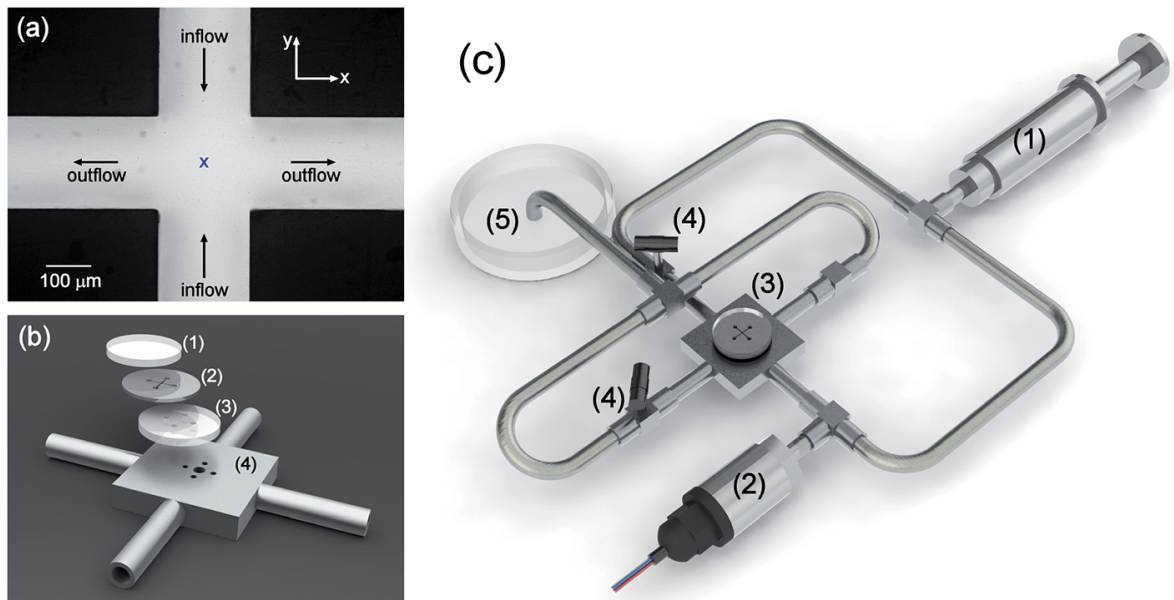

Fig. 2 Cross-slot extensional rheometry: (a) micrograph of a cross-slot, cut through $1 \mathrm{~mm}$ thick stainless steel by wire electrical discharge machining (wire-EDM). (b) Expanded schematic of a cross-slot flow-cell showing (1) glass front window, (2) stainless-steel cross-slot, (3) glass rear window with holes to permit fluid flow, and (4) stainless steel flow-cell mount with four tube connections to the flow system and a central "thru" hole to allow optical access to the stagnation point. (c) Illustration of the flow system showing (1) stainless-steel syringe (driven by a syringe pump), (2) pressure sensor (GE Druck), (3) cross-slot flow-cell, (4) needle valves to adjust the flow, and (5) Petri dish to receive effluent.

where $\operatorname{II}(\dot{\gamma})$ is the second invariant of the deformation rate tensor $\dot{\gamma}=\nabla \mathbf{u}+\nabla \mathbf{u}^{T}$. For the range of volume flow rates investigated we obtain a range of $0.03 \leq \mathrm{Re} \leq 40$, depending on the particular fluid.

Broadly speaking, elastic effects are expected to become significant as the Weissenberg number $(\mathrm{Wi}=\dot{\varepsilon} \lambda)$ exceeds unity, i.e. as material elements are being stretched faster than the dissolved polymers can relax. Hence, observation of elastic effects such as the onset of birefringence near the stagnation point or an enhanced pressure drop across the flow cell at a specific strain rate $\dot{\varepsilon}_{\mathrm{c}}$ indicates the coil-stretch transition ${ }^{\mathbf{9 , 5 4 , 8 7 - 9 0}}$ and can be used to estimate the relaxation time of the test fluid as $\lambda=1 / \dot{\varepsilon}_{\mathrm{c}}$.

The flow-induced birefringence arising in the fluid due to macromolecular orientation in the vicinity of the stagnation point is measured by using an ABRIO birefringence microscope system (CRi, Inc.). ${ }^{91,92}$ The cross-slot flow cell is placed on the imaging stage of an inverted microscope (Nikon Eclipse TE 2000-S) and the mid-plane of the flow cell is brought into focus using a $20 \times 0.5$ NA objective. Circularly polarized monochromatic light $(\lambda=546 \mathrm{~nm})$ is passed first through the sample, then through a liquid crystal compensator optical element and finally onto a CCD array. To acquire a flow birefringence image, five individual frames are captured with the liquid crystal compensator configured in a specific polarization state in each frame, and data from the five individual frames are converted into a full-field map of optical retardation and orientation angle. ${ }^{91,92}$ The system can measure the optical retardation $(R)$ of the polarized light to a nominal accuracy of $\sim 0.02 \mathrm{~nm}$, with a spatial resolution corresponding to a pixel size $\sim 0.5 \mu \mathrm{m}$ (with a $20 \times$ objective lens). The relationship between the retardation and the birefringence of the sample is given by $R=d \Delta n$, where $d$ is the depth of the flow cell.

By closing the two needle valves, labeled (4), the pressure drop can also be measured for steady viscous shearing flow of fluid around a single corner of the cross-slot $\left(\Delta P_{\text {shear }}\right)$. Subsequently, the pressure drop is measured with the two needle valves open $\left(\Delta P_{\text {total }}\right)$ to generate a stagnation point flow and the excess pressure drop $\left(\Delta P_{\text {excess }}=\Delta P_{\text {total }}-\Delta P_{\text {shear }}\right)$ arising as a result of the additional extensional component in the flow field is computed. It has previously been shown that the extensional stress difference $\Delta \tau$ in the stretching fluid is proportional to the excess pressure drop ${ }^{5,64}$ or $\Delta \tau \propto \Delta P_{\text {excess. }}$. We can therefore obtain a measure of the apparent extensional viscosity of the fluid thus:

$$
\eta_{\mathrm{E}, \mathrm{app}}\left(\dot{\varepsilon}_{\mathrm{CS}}\right) \approx \frac{\Delta P_{\text {excess }}}{\dot{\varepsilon}_{\mathrm{CS}}}=\frac{\Delta P_{\text {total }}-\Delta P_{\text {shear }}}{\dot{\varepsilon}_{\mathrm{CS}}}
$$

The Trouton ratio of the fluid is the ratio of the measured extensional viscosity to shear viscosity and is calculated from the definition $\operatorname{Tr}\left(\dot{\varepsilon}_{\mathrm{CS}}\right)=\eta_{\mathrm{E} \text {,app }}\left(\dot{\varepsilon}_{\mathrm{CS}}\right) / \eta(\dot{\gamma})$, where $\eta(\dot{\gamma})$ is the value of shear viscosity found from cone-and-plate rheometry and $\dot{\gamma}=2 \dot{\varepsilon}_{\mathrm{CS}}$, as described above. The expected Trouton ratio for Newtonian fluids undergoing planar elongational flow is $\operatorname{Tr}=4 .^{93}$

\subsection{Extensional rheometry based on capillary break-up dynamics}

In addition to the extensional viscosity measurements performed in the microfluidic cross-slot rheometer, we carried out capillary thinning and jetting measurements to identify and characterize the response of cellulose ether solutions to the rapid transient extensional deformations that arise in freesurface flows realized in actual jetting, printing and spraying processes. The underlying physics and the methods are described next.

2.3.1 Relevant forces, timescales and characteristic dimensionless groups. The pressure gradient created by a local region of high curvature in the neck of a filament connecting 
two larger drops drives a flow out of the neck resulting in additional thinning and eventually break-up or rupture. Capillary thinning and break-up can be observed in a number of prototypical geometries ${ }^{9,32}$ including: (I) dripping, where the pinch-off results from an interplay of gravitational drainage and capillarity, ${ }^{\mathbf{4 1}}$ (II) jetting, where convective instability develops on a fluid jet as it issues out of a nozzle, ${ }^{78}$ used in the ROJER measurements in this paper, and (III) self-thinning of a stretched liquid bridge as utilized in the Capillary Break-up Extensional Rheometer (CaBER). ${ }^{77}$

The CaBER technique enables the assessment of the longest molecular unraveling times and extensional stresses of viscoelastic fluids through monitoring of the capillary thinning and breakup dynamics of a fluid thread that is formed between two circular rigid end-plates. ${ }^{76,77}$ As the fluid neck thins locally over time under the action of capillary pressure, the dynamics of the thinning process depend upon the magnitude of the viscous, inertial and elastic stresses that oppose the pinch-off. The relative magnitudes of these four contributions to the stress, and the absence of external forcing, result in similarity solutions that determine the rate of thinning. ${ }^{9}$ The self-similar thinning dynamics can then be used to compute material parameters, such as the relaxation time for elastic fluids, as discussed in the next sub-section.

We now consider the capillary thinning dynamics in the jetting geometry used in the ROJER technique. An inviscid fluid jet issuing out of a nozzle is unstable to a range of different perturbations. The characteristic growth rate, $\alpha$, for a perturbation of wavelength, $\lambda_{\mathrm{p}}$ (or wavenumber, $k=2 \pi / \lambda_{\mathrm{p}}$ ), is given by an expression first derived and discussed by Rayleigh ${ }^{\mathbf{9 4 - 9 6}}$ to be

$$
\frac{\alpha}{\alpha_{0}}=x\left(1-x^{2}\right) \frac{I_{1}(x)}{I_{0}(x)}
$$

here $x=k R_{0}$ is the wavenumber non-dimensionalized by the radius of the nozzle, such that $0 \leq x \leq 1$ and $I_{j}(x)$ represent the Bessel function of order $j$. Here the velocity and length scales are set by the flow rate, $Q$, and the size of the nozzle, $D_{0}=2 R_{0}$. The growth rate in eqn (3) is non-dimensionalized by $\alpha_{0}$, which is the inverse of the Rayleigh time $t_{\mathrm{R}}$ (the timescale characterizing inviscid drop oscillations) and is defined as

$$
t_{\mathrm{R}}=\alpha_{0}{ }^{-1}=\left(\frac{\rho R_{0}{ }^{3}}{\sigma}\right)^{1 / 2}
$$

here $\rho$ and $\sigma$ are the density and surface tension of the fluid respectively. A free jet breaks under the fastest growing instability. The maximum growth rate can be computed from the peak of the dispersion curve (using eqn (3)). Pertubations with the maximum growth rate, $\alpha_{\max }=0.34 \alpha_{0}$ appear at $x_{\max }=$ $k_{\max } R_{0}=0.69$. The break-up length, $L_{\mathrm{B}}$, of the jet is predicted by linear stability analysis to be shortest for the frequency $k_{\max }$ at which the maximum growth rate $\alpha_{\max }$ is achieved. The break-up length $L_{\mathrm{B}}$ of inviscid or low viscosity Newtonian fluid jets ${ }^{97}$ is linearly proportional to the jet velocity, $V_{\mathrm{j}}$, and can be written as $L_{\mathrm{B}} / D_{0}=C \mathrm{We}^{0.5}$. Here, the Weber number, We $=\mathrm{ReCa}=$ $\rho V_{\mathrm{j}}^{2} D_{0} / \sigma$ is the dimensionless ratio of the stagnation pressure in the jet to the capillary pressure (which acts to break the jet into drops) and typically the constant $C \approx 12$. The presence of viscous effects changes both the dispersion curve and the breakup length ${ }^{97}$ to an expression of the form $L_{\mathrm{B}} / D_{0}=$ $C \mathrm{We}^{0.5}(1+3 \mathrm{Oh})$. Here the Ohnesorge number, which contrasts the relative importance of viscosity to inertio-capillary effects, is defined as $\mathrm{Oh}^{-2}=\mathrm{Re} / \mathrm{Ca}=\rho \sigma D / \eta_{0}{ }^{2}$. The Ohnesorge number scales linearly with viscosity and does not depend upon the jet velocity. In contrast to free jets, for a harmonically vibrated jet, the dominant perturbation on the jet is selected by the choice of perturbation frequency, $f_{\mathrm{p}}$, and the velocity of the jet, $V_{\mathrm{j}}$ (so that wavenumber, $k=2 \pi f_{\mathrm{p}} / V_{\mathrm{j}}$ ). In this case, the break-up length can be measured directly as a function of imposed perturbation frequency, and for low viscosity fluids, the shortest break-up length corresponds to the wavenumber $x_{\max }=k_{\max } R_{0}=0.69$.

The break-up of non-Newtonian or viscoelastic jets is markedly different from viscous jets because elastic effects are typically important. The relative contribution of elastic and viscous effects is characterized through an elasticity number, $\mathrm{El}=\eta_{0} \lambda / \rho D_{0}{ }^{2}$, which does not depend upon the process kinematics. Since the elasticity number depends inversely on the square of the length scale, viscoelastic effects are bound to become more significant with any decrease in the diameter of a filament undergoing thinning. For weakly elastic jets, where $\mathrm{El} \ll(1+3 \mathrm{Oh})^{2}$, Middleman ${ }^{98}$ used linear stability analysis to show

$$
\frac{L_{\mathrm{B}}}{D}=\frac{C \mathrm{We}^{0.5}(1+3 \mathrm{Oh})}{\left(1+3 \mathrm{El} /(1+3 \mathrm{Oh})^{2}\right)}
$$

This expression suggests that the break-up length is lower for weakly elastic fluids. Furthermore linear stability analysis shows that viscoelastic jets initially have faster disturbance growth than a Newtonian fluid of the same viscosity. ${ }^{\mathbf{9 8 - 1 0 3}}$ However, in the case of strongly strain-hardening polymer solutions, the capillarity-driven thinning is opposed at large strains by the presence of an extra stress due to polymer stretching, and this can subsequently stabilize the jet against pinch-off. ${ }^{33,37,38,99,101}$ In some cases, the build-up of extra elastic stresses during filament thinning can lead to the development of extremely stable beads-on-a-string morphologies, and consequently lead to much longer break-up lengths. ${ }^{39,43}$

Experimentally, the delay in pinch-off due to contributions of elastic stresses can be used to determine the apparent extensional viscosity, ${ }^{\mathbf{9 , 4 0 , 1 0 4}}$ and this underlies the basis for both CABER and ROJER techniques. In the elasto-capillary regime, the transient extensional properties of the fluid can be determined by measuring the rate of evolution in the mid-filament diameter and by using the balance between elastic and capillary forces, as described in eqn (6)-(9) below. For a cylindrical fluid filament, we can define the instantaneous strain rate $(\dot{\varepsilon})$ and the accumulated Hencky strain $\left(\varepsilon_{\mathrm{H}}\right)$, as follows: ${ }^{42,104}$

$$
\begin{gathered}
\dot{\varepsilon}(t)=-\frac{2}{D(t)} \frac{\mathrm{d} D(t)}{\mathrm{d} t} \\
\varepsilon_{\mathrm{H}}(t)=\int_{0}^{t} \dot{\varepsilon}(t) \mathrm{d} t=2 \ln \left(\frac{D_{0}}{D(t)}\right)
\end{gathered}
$$


where $D_{0}$ is the initial diameter of the filament at $t=0$. The axial force on a cylindrical thinning fluid column is given by the following stress balance:

$$
\Delta \tau(t)=3 \eta_{\mathrm{s}} \dot{\varepsilon}(t)+\left(\tau_{\mathrm{zz}}-\tau_{\mathrm{rr}}\right)=\frac{2 \sigma}{D(t)}
$$

where $2 \sigma / D(t)$ is the capillary pressure driving the filament thinning process and $\Delta \tau(t)$ is the total extensional stress difference in the elongating filament. Combining eqn (6) and (8), the apparent transient extensional viscosity of the stretching fluid can then be evaluated using the following expression:

$$
\eta_{\mathrm{E}}=\frac{\Delta \tau(t)}{\dot{\varepsilon}(t)}=-\frac{\sigma}{\mathrm{d} D(t) / \mathrm{d} t}
$$

For an aqueous polymer solution such as the cellulosic system studied in the present work, Entov and Hinch ${ }^{\mathbf{1 0 4}}$ argued that in the elasto-capillary thinning regime, the mid-plane diameter of the filament decays exponentially as

$$
\frac{D(t)}{D_{0}}=\sum_{i}\left(\frac{g_{i} D_{0}}{4 \sigma}\right)^{1 / 3} \exp \left[-t / 3 \lambda_{i}\right] \approx\left(\frac{g D_{0}}{4 \sigma}\right)^{1 / 3} \exp [-t / 3 \lambda]
$$

where $g$ is the elastic modulus and $\lambda$ is the longest fluid relaxation time, both of which will vary with cellulose concentration. The complete solution requires a consideration of the whole relaxation spectrum, where $g_{i}$ and $\lambda_{i}$ are respectively the modulus and relaxation time contributed by each mode $i$. However, for long times the self-similar thinning process means that only the longest mode is important. The relative importance of elastic effects in a jet is characterized by a dimensionless group, called the intrinsic Deborah number, De $=\lambda / t_{R}$, which represents the ratio of the longest relaxation time of the fluid, $\lambda$, to the Rayleigh time scale, $t_{\mathrm{R}}$. Polymer molecules undergoing sustained extension can eventually approach the fully stretched state and reach the finite extensibility limit. Beyond this point eqn (10) becomes invalid and the radius eventually decays linearly with time. ${ }^{9}$ However due to the presence of fully extended polymer chains, the pinch-off dynamics can be significantly slower than the behavior exhibited by Newtonian fluids. Finite extensibility effects and the scaling relationship between radius and time that capture the pinch-off dynamics are discussed in a subsequent section.

2.3.2 Capillary break-up extensional rheometer (CaBER). The extensional properties of cellulose ether solutions were first tested using a Capillary Breakup Extensional Rheometer (CaBER; Cambridge Polymer Group) to impose a predominantly uniaxial extensional deformation to the fluid samples. The CaBER device uses an initially cylindrical volume of fluid $(V \approx 0.06 \mathrm{~mL})$, which forms a liquid bridge between circular parallel plates of diameter $D_{0}=6 \mathrm{~mm}$ and initial separation $L_{0}=2 \mathrm{~mm}$ (initial aspect ratio $\Lambda_{0}=L_{0} / D_{0}=0.33$ ). To minimize gravitational sagging and obtain an approximately cylindrical liquid bridge, the initial separation was chosen to be less than the capillary length $l_{\text {cap }}=\sqrt{\sigma / \rho g}$, where $\sigma$ is the surface tension, $\rho=1.0 \mathrm{~g} \mathrm{~cm}^{-3}$ is the solvent (water) density, and $g=9.81 \mathrm{~m} \mathrm{~s}^{-2}$ is the acceleration due to gravity. ${ }^{77}$ The surface tension of the aqueous cellulose ether solutions was measured to be $\sigma=60 \mathrm{mN} \mathrm{m}^{-1}$ using a Krüss K10ST digital tensiometer, resulting in $l_{\text {cap }} \approx 2.2 \mathrm{~mm}$.

At time $t=-50 \mathrm{~ms}$ the top endplate was displaced upwards following an exponential profile $L(t)=L_{0} \mathrm{e}^{\dot{\varepsilon}_{0} t}$ to achieve a final plate separation of $L_{\mathrm{f}}=6 \mathrm{~mm}$ at time $t=0 \mathrm{~s}$ (final aspect ratio $\Lambda_{\mathrm{f}}=L_{\mathrm{f}} / D_{0}=1.0$ ). The subsequent evolution of the liquid filament diameter $(D(t))$ was monitored at the midplane between the endplates (i.e. at $\left.L=L_{\mathrm{f}} / 2\right)$ using a laser micrometer. The dynamics of the liquid bridge thinning and break-up process were also recorded at 60 frames per second using a 6 megapixel CCD camera (Casio Exilim EX-F1), with a resolution of approximately $7 \mu \mathrm{m}$ per pixel. In uniaxial extensional flow Newtonian fluids (with rate-independent shear viscosity) display an extensional viscosity that is three times the shear viscosity. Thus for Newtonian fluids, the ratio of extensional viscosity to shear viscosity, defined as the Trouton ratio is $\operatorname{Tr}=\eta_{\mathrm{E}} / \eta_{0}=3$.

2.2.3 Rayleigh Ohnesorge Jetting Extensional Rheometry (ROJER). The jetting rheometry technique described here was developed specifically to study weakly viscoelastic fluids such as cellulose ether solutions. ${ }^{70,71}$ In the jetting rheometer, the spontaneous break-up of a fluid jet issuing from a small nozzle is controlled by imposing perturbations of known frequency, $f_{\mathrm{p}}$, and amplitude. Axisymmetric sinuous perturbations are created on a fluid jet by using a piezoelectric transducer that imposes radial pressure onto the nozzle. The perturbation amplitude and frequency are controlled and set by a function generator. The configuration for forced jetting experiments includes a ceramic nozzle of diameter $2 R_{0}=175$ microns mounted on a precision stage, so that the position of the nozzle and vertical alignment can be controlled using screw micrometers. The jet is illuminated using a strobe-backlight, where the strobe frequency is either synchronized to the driving frequency, $f_{\mathrm{p}}$, of the piezoelectric transducer or maintained at a known variation, $\Delta f_{\mathrm{p}}$, from it using a second function generator. If the perturbation frequency, $f_{\mathrm{p}}$, is out of sync with the strobe frequency, i.e. $\Delta f_{\mathrm{p}}>0$, the growth and evolution of the instability of the jet can be observed in an apparent slow motion. The apparent velocity of the jet, $u_{\text {app }}$, as observed using a strobe frequency, $f_{\mathrm{p}}-\Delta f_{\mathrm{p}}$, is related to the flow rate, $Q$, and the radius of the nozzle by the following equation:

$$
M u_{\mathrm{pix}} F=u_{\mathrm{app}}=\frac{\Delta f_{\mathrm{p}}}{f_{\mathrm{p}}} \frac{Q}{\pi R_{0}{ }^{2}}
$$

The first equality relates the apparent velocity $u_{\text {app }}$ to the parameters from the image analysis, where $M$ is the magnification (related to the ratio of nozzle size in microns to its size in pixels), $u_{\text {pix }}$ is a velocity defined in terms of pixels moved per frame, and $F$ is the frame rate. The second equality describes the stroboscopic effect. The higher the driving frequency, the greater is the apparent slowing down of the jet. Typical operating frequencies are in the range of a few $\mathrm{kHz}$, the field of view ranges from $0.5 \mathrm{~mm}$ to a few $\mathrm{mm}$, and the typical observable feature size is in the size range of 5-200 $\mu \mathrm{m}$. For a driving frequency of $f_{\mathrm{p}}=4 \mathrm{kHz}$ and a frequency delay of $\Delta f_{\mathrm{p}}=0.1 \mathrm{~Hz}$, the fluid is observed at 40000 times slower speed, allowing us to resolve the dynamics of jet thinning in a low viscosity complex 
fluid using conventional video imaging hardware. A chargecoupled device (CCD) camera (BlueFox, Matrix Vision) with an attached zoom lens collects the images at a rate of around $F=$ $40 \mathrm{fps}$, and the movies are stored in digital, unprocessed form on an external hard-disk connected to the computer. Some of the jetting experiments are alternatively acquired using a JetXpert (ImageXpert, Nashua, NH) imaging system, where the frame rate is restricted to $6 \mathrm{fps}$. Air pressure or a precision syringe pump (Harvard PhD Ultra) is used to control the flow rate but the precision required in the uniformity of the flow rate limits us to fluids with viscosity below $20 \mathrm{mPa}$ s. Image analysis is carried out using Image ${ }^{\mathbf{1 0 5}}$ (an imaging program developed by NIH, available freely), and custom programs specifically written for this purpose in MATLAB (Mathworks, Natick, MA). The thinning dynamics are quantified by using a MATLAB program that follows the material deformation of a Lagrangian fluid element as it moves along the jet.

\section{Results and discussion}

\subsection{Cross-slot flow extensional rheometry}

In Fig. 3 we present images of the flow-induced birefringence observed near the stagnation point of the cross-slot for solutions of EHEC and hmEHEC over a representative range of strain rates. The flow-induced birefringence provides a measure of the segmental anisotropy of macromolecules as they deform and align in the flow field and thus provides an indicator of the degree of macromolecular strain..$^{51,106,107}$ Qualitatively all of the fluids display similar behavior as the strain rate is increased. At low strain rates, a narrow birefringent strand is observed that is highly localized along the central outflow axis passing through the stagnation point. Macromolecules that follow fluid elements along this streamline are the first to stretch since they experience the highest fluid strain. As the strain rate is increased the magnitude of the birefringence also increases and, while remaining quite localized, the birefringent strand grows in length and breadth. Similar responses are observed in PEO solutions and in worm-like micellar solutions. ${ }^{\mathbf{5 , 6 4 , 6 6}}$ The birefringence is greater in the more concentrated solutions, as expected.

Fig. 4 provides a quantitative measure of the maximum birefringence (assessed at the stagnation point) as a function of the imposed strain rate for all six fluids. We find that the birefringence increases gradually, starting from very low extensional strain rates and approaches plateau values at high rates. This behavior is typical of that expected for rigid or semi-rigid macromolecules such as cellulose-based polymers, which readily align in extensional flow fields. One notable difference between the data from the EHEC and the hmEHEC solutions occurs at the $1 \%$ concentration, where a much higher birefringence is recorded in the EHEC at high deformation rates. We note that for $\dot{\varepsilon}_{\mathrm{CS}}<300 \mathrm{~s}^{-1}$ both fluids show a similar response, however for $\dot{\varepsilon}_{\mathrm{CS}}>300 \mathrm{~s}^{-1}$ the birefringence in the hmEHEC increases more slowly than in the EHEC, due to the sticker-mediated microstructural changes in the hmEHEC solutions. The rheo-optical responses of the multisticker hmEHEC polymer dispersion and the bare EHEC dispersions both show a large absolute value of birefringence. High degrees (i)

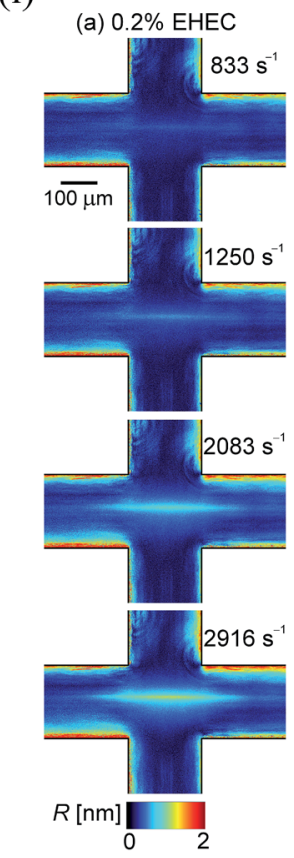

(b) $0.4 \%$ EHEC

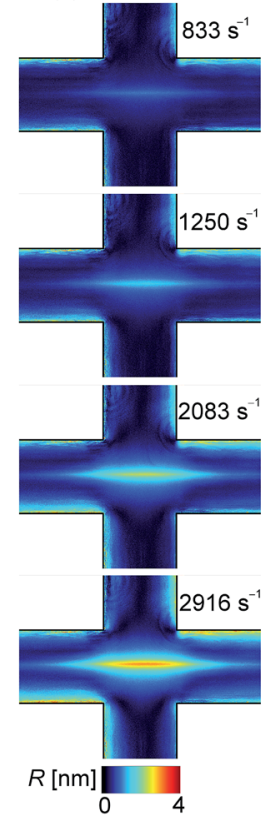

(c) $1.0 \%$ EHEC

(ii)

(a) $0.2 \% \mathrm{hmEHEC}$

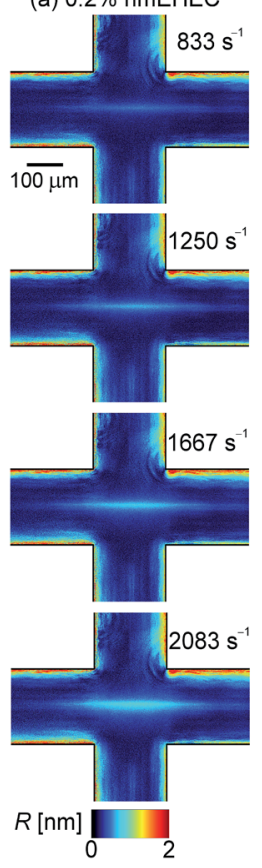

(b) $0.4 \%$ hmEHEC

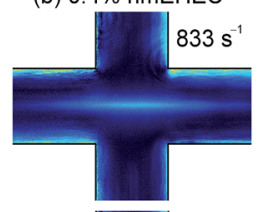

(c) $1.0 \% \mathrm{hmEHEC}$
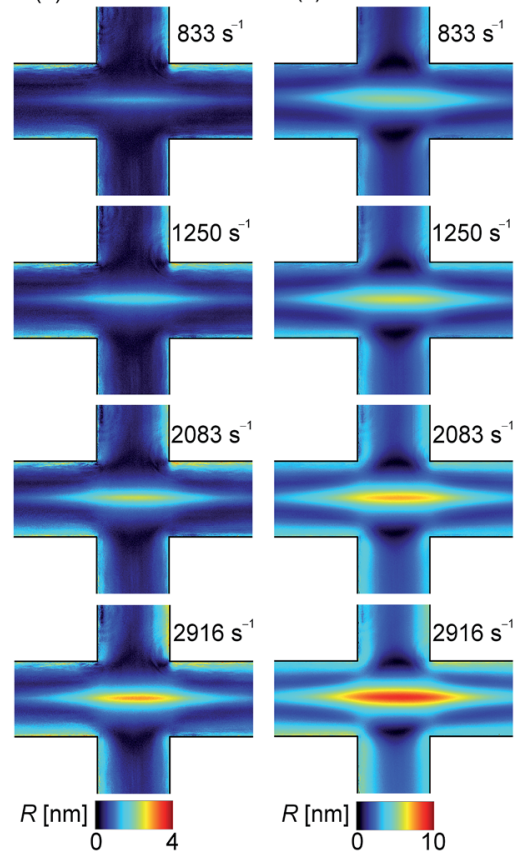

Fig. 3 (i) Birefringence in EHEC aqueous solutions in a cross-slot flow at the extensional rates indicated. Scale bar represents (a) 0-2 $\mathrm{nm}$, (b) 0-4 nm, and (c) 0-20 nm. (ii) Birefringence in hmEHEC aqueous solutions in a cross-slot flow at the extensional rates indicated.

of chain stretching at the stagnation point for $\dot{\varepsilon}_{\mathrm{CS}}>1 / \lambda$ have been demonstrated in cross-slot geometries by studies of flow induced birefringence ${ }^{51,64,106-108}$ and by direct observation of fluorescently labeled DNA. ${ }^{7,109,110}$ This stretching has been shown to coincide with a significant increase in the pressure drop measured across the cross-slot, consistent with the predicted increase in the extensional viscosity. ${ }^{51,64,106-108}$ 

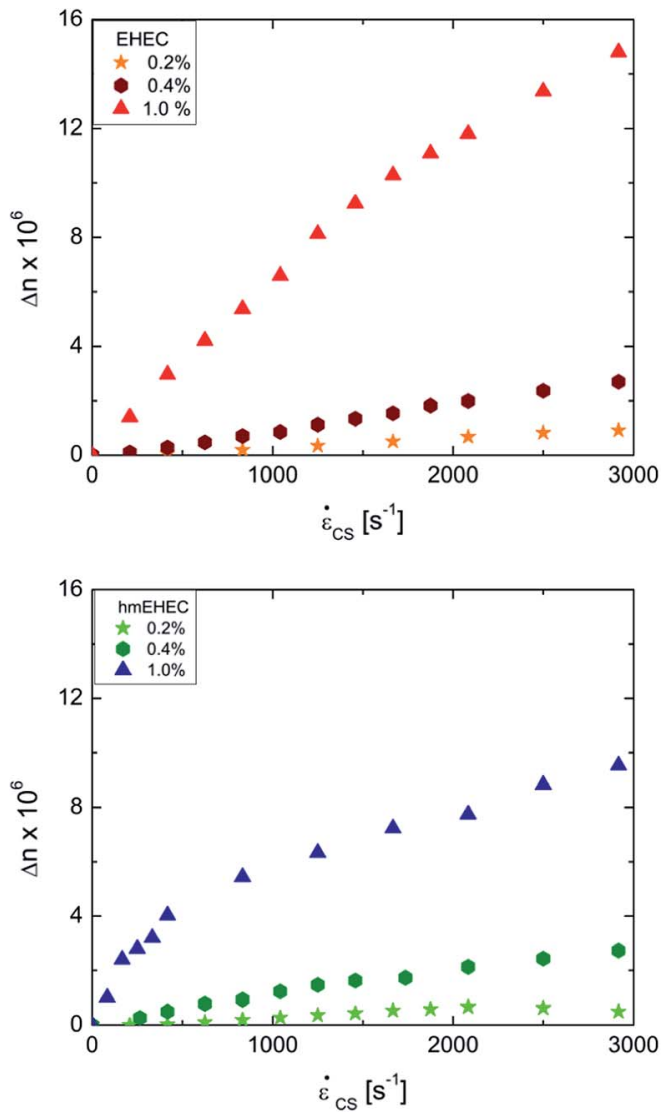

Fig. 4 Flow birefringence as a function of imposed strain rate, measured in the cross-slot extensional rheometer. Top: EHEC dispersion, bottom: hmEHEC dispersions.

In Fig. 5, we show the measured extensional flow birefringence versus excess pressure drop data For EHEC solutions. The optical birefringence from macromolecular stretching is linearly proportional to the excess pressure drop measured at low extensional rates, and this provides an estimate of the stressoptical coefficient, $C_{\mathrm{SOR}}$, as the fluid appears to obey the stress optical rule $\Delta n=C_{\mathrm{SOR}} \Delta \tau$. The estimated value for the stress-optical coefficient from the data in Fig. 5 is $C_{\text {SOR }}=2.8 \times$ $10^{-8} \mathrm{~Pa}^{-1}$. Also shown in Fig. 5 are measurements of extensional flow birefringence versus excess pressure drop data obtained in the same geometry for two flexible polymers, polyethylene oxide in water and polystyrene in DOP, an aqueous solution of wormlike micelles and an aqueous solution of hyaluronic acid (HA), which is also a polysaccharide. The measured stress-optical coefficient for the EHEC solution is comparable to values known for other polysaccharides. For example, for HA in PBS solution $C_{\mathrm{SOR}}=1.82 \times 10^{-8} \mathrm{~Pa}^{-1}$ was reported by Kulicke and coworkers ${ }^{111}$ while Haward et al. reported $C_{\mathrm{SOR}}=5.9 \times 10^{-8}$ $\mathrm{Pa}^{-1}$ using cross-slot extensional rheometry measurements. ${ }^{5}$ Kulicke et al. ${ }^{112}$ have reported concentration-dependent values $2 \times 10^{-8} \mathrm{~Pa}^{-1}<C_{\mathrm{SOR}}<8 \times 10^{-8} \mathrm{~Pa}^{-1}$ for sodium carboxymethyl cellulose. The values of flow birefringence and corresponding optical anisotropy of polymer segments in solutions of cellulose derivatives are noticeably greater than for the solutions of flexible chain polymers. The data shown in Fig. 5 clearly

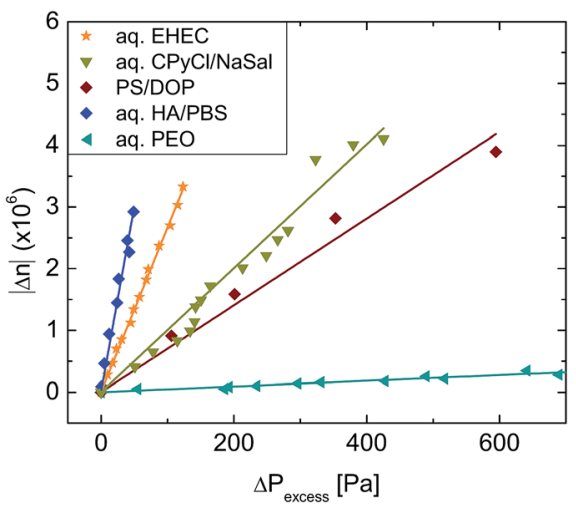

Fig. 5 Stress optical diagram. The flow birefringence measured in the cross-slot device plotted as a function of excess pressure drop (see text for details on fluids compared). The slope of each line gives the stress-optical coefficient $C_{\text {SOR }}$ for each fluid.

illustrate that the EHEC segments align in the direction of flow quite easily and the behavior qualitatively illustrates the fact that the chains behave as semi-rigid polymers.

In Fig. 6a we present the apparent extensional viscosity as a function of the strain rate for the EHEC solutions in the crossslot device. The extensional viscosity is derived from pressure drop measurements using eqn (1) and (2) and the Trouton ratio is computed using $\operatorname{Tr}\left(\dot{\varepsilon}_{\mathrm{CS}}\right)=\eta_{\mathrm{E} \text {,app }}\left(\dot{\varepsilon}_{\mathrm{CS}}\right) / \eta(\dot{\gamma})$. The corresponding raw pressure data are presented in the ESI. $\dagger$ At low flow rates, the pressure due to the extensional component of the flow field is computed as a difference of two small numbers, and hence the error bars in the low deformation rate region are relatively high. At the lowest concentration the apparent extensional viscosity is approximately constant at $\eta_{\mathrm{E}, \mathrm{app}} \approx 0.01 \mathrm{~Pa} \mathrm{~s}$, which equates to a Trouton ratio ( $\mathrm{Tr}$ ) very close to the expected value for Newtonian fluids in planar extensional flow of $\mathrm{Tr}=4$ (Fig. 6b). As the EHEC concentration is increased we observe a non-Newtonian increase in the extensional viscosity, starting from very low strain rates, and settling to plateau values at strain rates beyond $2000 \mathrm{~s}^{-1}$. The extensional viscosity increase provides a significant increase in the Trouton ratio to $\mathrm{Tr} \approx 10$ and $\mathrm{Tr}>20$ for the $0.4 \%$ and $1.0 \%$ EHEC solutions, respectively. A similar plateau in extensional viscosity of hydroxypropyl-ether guar gum solutions was reported previously by Duxenneuner et al. ${ }^{113}$

The apparent extensional viscosity and Trouton ratio data for the hydrophobically modified hmEHEC solutions are shown in Fig. 7a and b, respectively. We observe markedly different behavior from that seen with the corresponding EHEC solutions. At the lower concentrations of hmEHEC, we observe apparent extensional thinning behavior as the strain rate is increased. The Trouton ratio for these fluids initially (at low extension rates) exhibits fairly high values of around $\mathrm{Tr} \approx 56$ and $\mathrm{Tr} \approx 40$ for the $0.2 \%$ and $0.4 \%$ solution respectively. However, the Trouton ratio of both fluids drops to a low plateau value of around 10 at a strain rate of about $1000 \mathrm{~s}^{-1}$. In contrast, the $1.0 \%$ hmEHEC solution displays an initially increasing apparent extensional viscosity, which subsequently decreases for strain rates $\dot{\varepsilon}_{\mathrm{CS}}>10^{3} \mathrm{~s}^{-1}$. This equates to a Trouton ratio that initially rises from a value $\operatorname{Tr} \approx 3$ up to a maximum of around 10 , followed by a rapid reduction. 
(a)

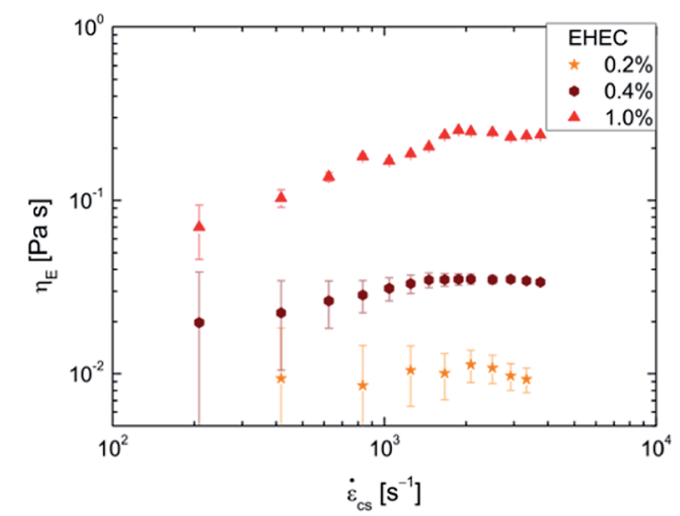

(b)

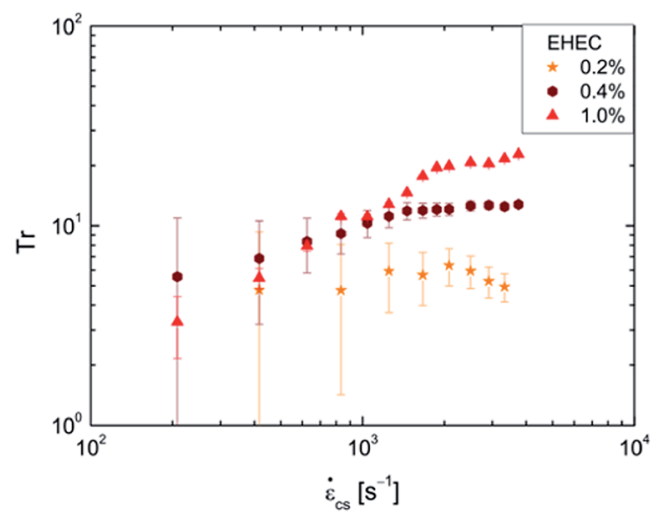

Fig. 6 Cross-slot extensional rheometry for EHEC solutions. (a) Extensional viscosity and (b) Trouton ratio as a function of imposed strain rate, determined from measurements in the cross-slot extensional rheometer for three EHEC solutions.

Similar extensional thinning in associative polymers with stickers distributed along the chain was also seen in studies of a hydrophobically modified alkali soluble associative (HASE) system by Tan et al. ${ }^{114}$ Solutions of associating HASE polymers with C-12 (12 carbon long) hydrophobic stickers showed extensional thickening at intermediate extension rates followed by a large extensional thinning at the highest rates that could be achieved using an opposed-jet rheometer. In their study, the bare hydrophilic chain exhibited a plateau value in extensional viscosity at high extension rates, qualitatively similar to our observations for EHEC solutions. These authors also studied the effect of increasing the strength of association by comparing C-12 with C-16 and C-20 modified chains and determined that chains with stronger intermolecular association show only extensional thinning as a result of the progressive disruption of the microstructure at high deformation rates.

The physics underlying extensional thinning of multisticker polymer dispersions in response to strong elongation flows can be broadly understood by using arguments from nonlinear network models developed for describing the steady and transient shear and extensional response of associative polymer solutions, including for telechelic polymers in which hydrophobic stickers are present only at the chain ends. ${ }^{22,25,115}$ In a (a)

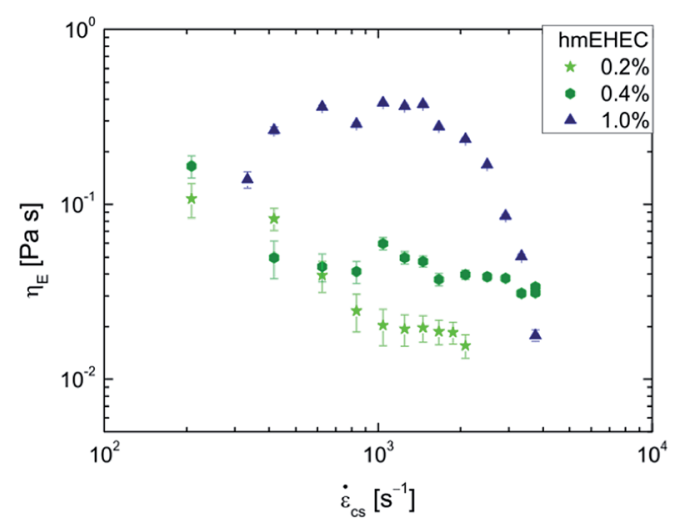

(b)

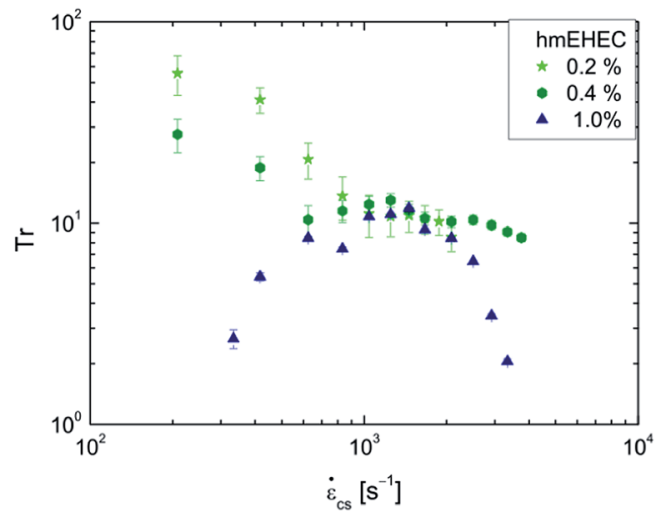

Fig. 7 Cross-slot extensional rheometry for hmEHEC solutions. (a) Extensional viscosity and (b) Trouton ratio as a function of imposed strain rate, determined from pressure drop measurements in the cross-slots for three hmEHEC solutions.

quiescent state, the temporary associations are formed and destroyed with a disassociation time, $\lambda_{\mathrm{do}} \approx \Omega^{-1} \exp \left(\Delta G / k_{\mathrm{B}} T\right)$ which depends on the activation energy $\Delta G$ that in turn depends upon the number of carbon atoms (or methylene groups) present in the hydrophobic group. Under strong extensional flow conditions, the sticky chains are stretched and aligned which leads to an anisotropic enhancement in the frequency and duration of encounters between hydrophobic stickers as well as resulting in an enhanced stretching or pullout force on the stickers that acts to break down the transient junctions. As the hmEHEC solutions are exposed to an extensional flow field, the data in Fig. 7 show that the chain alignment and stretching result in an increase in the number of associations between the bridging segments and consequently to an extensional thickening at intermediate rates. A further increase in extension rate and the onset of finite chain extensibility effects leads to a breakdown of the physical network structure and correspondingly to a large extensional thinning. In addition to associative polymers where break-up of the transient network structure leads to a decrease in extensional viscosity, extensional thinning is also observed in dilute and semi-dilute polymer solutions in experiments $\mathrm{s}^{84,116-118}$ as well as simulations..$^{88,118,119} \mathrm{In}$ such cases, the extensional thinning can be described using 
constitutive models like the Wiest model ${ }^{85}$ and the Primitive Chain Network model ${ }^{\mathbf{8 1}, \mathbf{8 2}}$ that incorporate finite extensibility along with anisotropic hydrodynamic drag. ${ }^{\mathbf{8 1 - 8 3 , 8 5 , 1 2 0}}$

The concentration-dependent birefringence measured at a given extension rate represents the effective orientation and stretching of macromolecules in EHEC and hmEHEC solutions in response to the extensional deformation, in addition to the competition between relaxation of chains and their deformation. Rescaling the birefringence with the polymer concentration, and also rescaling the extension rate by a critical extension rate, enables the data to be superimposed as shown in Fig. 8. The scaled birefringence or specific extensional flow birefringence $\Delta n /\left(c^{\prime} / \rho\right)$ captures the average conformational anisotropy, while the rescaled extension rate $\dot{\varepsilon} / \dot{\varepsilon}_{\mathrm{c}}$ can be considered to be equivalent to an effective Weissenberg number Wi, which provides a measure of the flow strength. Here the birefringence $\Delta n$ is scaled with a dimensionless ratio of concentration $c^{\prime}$ and density, $\rho$ (calculated with mass/volume units). The inset of Fig. 8a also displays extensional stress scaled by modulus, $\tau_{\mathrm{E}} / G_{\mathrm{N}}=\tau_{\mathrm{E}} / c^{\prime} R T$ for EHEC solutions, as a function of the effective Weissenberg number, and the extensional response from different EHEC solutions is remarkably similar. The superposition of normalized extensional stress and effective Weissenberg number shows that the observed nonlinear response arises from macromolecules that align and stretch in a self-similar fashion in response to applied deformation of similar strength. Similar plots of specific birefringence defined as $\Delta n / c^{\prime}$ and reduced extension rate defined as $r=\dot{\varepsilon} / \dot{\varepsilon}_{\mathrm{c}}-1$ have also been presented by Sasaki et $a{ }^{121}$ for collagen dispersions (rod-like polymers) and Fujii et $a{ }^{\mathbf{1 2 2}}$ for semi-dilute solutions of hydroxypropylcellulose in water-glycerol mixtures (semi-flexible polymers). The scaling carried out here for extensional flow-induced birefringence is similar in principle to the plots of specific birefringence versus reduced extension rate for dilute solutions, ${ }^{5,51}$ where $\dot{\varepsilon}_{\mathrm{c}}=1 / \lambda_{\mathrm{cs}}$ is based on the relaxation time for coil-stretch transition. Similarly the specific birefringence measured in response to shear flow of polydisperse semi-flexible and rodlike polymers in solution overlaps with each other when plotted as a function of shear rate normalized by the rotational diffusion coefficient. ${ }^{123,124}$

The superposition that follows from rescaling the birefringence by concentration suggests that the underlying fundamental contributions to the observed birefringence arise from conformational changes of individual macromolecules in EHEC dispersions. By contrast, the specific birefringence of hmEHEC dispersions in Fig. 8b exhibits a lower slope at low extension rates and the scaled data for $c=1 \%$ hmEHEC dispersion show a much lower specific birefringence when compared to the corresponding EHEC solutions. This suggests the onset of interchain effects (arising from hydrophobic stickers) that limit the molecular extension and result in the decrease in the extensional viscosity observed in Fig. $7 \mathrm{~b}$.

\subsection{Capillary break-up extensional rheometry of cellulose ether dispersions}

In the Capillary Break-up Extensional Rheometer (CaBER) experiment, $2 \%$ by weight aqueous dispersions of EHEC and (a)

(b)
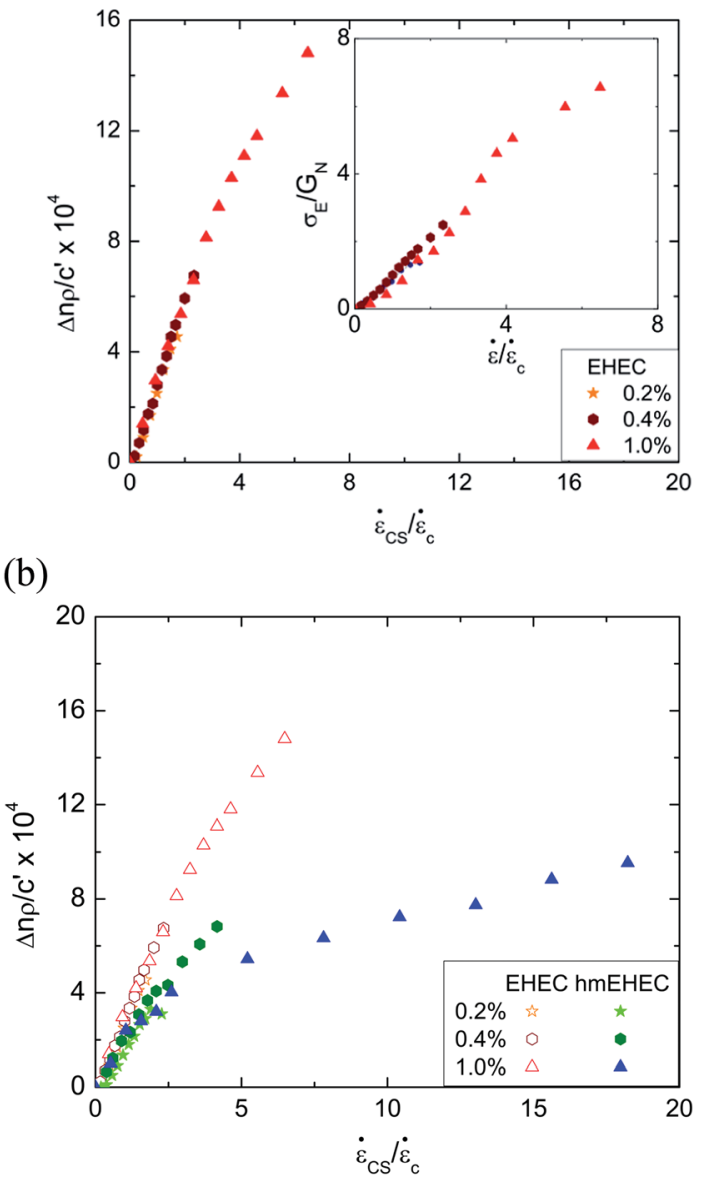

Fig. 8 Rescaled birefringence plotted as a function of rescaled extension rate (a) EHEC solutions, with the inset showing rescaled extensional stress vs. extension rate (b) hmEHEC dispersions, where EHEC data are shown to highlight the difference in conformational anisotropy.

hmEHEC both exhibit a linear decrease in filament radius with time as shown in Fig. 9. A linear decrease in radius with time suggests that a visco-capillary response, visible when $\mathrm{Oh} \sim \mathrm{O}(1)$, is observed here. This viscocapillary response can be described by the following expression: ${ }^{9,125}$

$$
\frac{D(t)}{D_{0}}=0.14 \frac{\sigma}{D_{0} \eta_{\mathrm{eff}}}\left(t_{\mathrm{p}}-t\right)=0.14 \mathrm{Oh}^{-1}\left(\frac{t_{\mathrm{p}}-t}{t_{\mathrm{R}}}\right)
$$

where $\mathrm{Oh}=\eta_{0} / \sqrt{\rho \sigma D_{0}}$ is the Ohnesorge number and the numerical pre-factor is found from the solution for the selfsimilar shape of capillary-driven thinning of a viscous fluid filament. ${ }^{125}$ Using the zero shear viscosity of EHEC $\eta_{0}=0.26 \mathrm{~Pa}$ s and hmEHEC $\eta_{0}=1.85 \mathrm{~Pa} \mathrm{~s}$, the estimates for the Ohnesorge number $(\mathrm{Oh})$ are $\mathrm{Oh}=0.5$ and $\mathrm{Oh}=3.6$ respectively. The longer pinch-off time, $t_{\mathrm{p}}$, for the hmEHEC dispersion $\left(t_{\mathrm{p}}=0.33 \mathrm{~s}\right)$ compared with EHEC solution $\left(t_{\mathrm{p}}=0.08 \mathrm{~s}\right)$ is due to the higher shear viscosity of the hydrophobically modified polymer dispersion. The slope of the radius versus time plots shown in Fig. 9 can be used to estimate the effective shear viscosity, and for viscous, Newtonian fluids, the estimated viscosity is within 


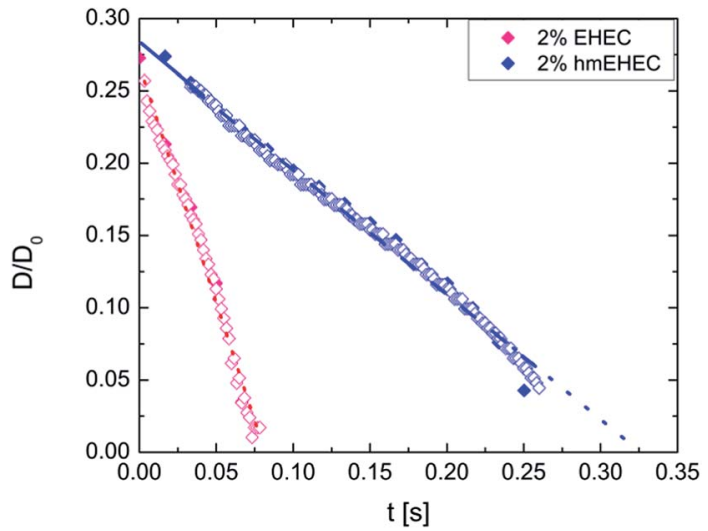

Fig. 9 Capillary thinning analysis of 2 wt\% cellulose ether solutions. Diameter vs. time measured using a laser micrometer is shown in filled symbols for $2 \%$ EHEC solution and $2 \%$ hmEHEC solution. The open symbols represent diameter vs. time data obtained from image analysis.

$5 \%$ of the values obtained in shear measurements. ${ }^{125}$ However, in the present experiments, the slopes correspond to $\eta_{\text {eff }}=$ $0.021 \mathrm{~Pa} \mathrm{~s}$ (i.e. $\mathrm{Oh}=0.04$ ) for EHEC and $\eta_{\mathrm{eff}}=0.08 \mathrm{~Pa} \mathrm{~s}$ (i.e. $\mathrm{Oh}=0.16)$ for hmEHEC dispersions, implying that the effective solution viscosity during capillary thinning is an order of magnitude lower than the zero shear viscosity. Both solutions are highly shear thinning and the lower effective viscosity is a result of this deformation rate-dependent viscosity. The presence of associating hydrophobic stickers in hmEHEC dispersions as well as the possibility of association through hydrogen bonding and hydrophobic side-groups in EHEC solutions provides these dispersions with a complex microstructure. The initial deformation of the sample required to generate the liquid bridge in the CaBER device leads to microstructural changes that result in lower values of the effective solution viscosity. A similar effect of initial step-strain is also observed for wormlike micelles and immiscible polymer blends; ${ }^{126,127}$ however the measurements for dilute polymer solutions are found to be insensitive to the amplitude and rate of step strain.

The typical concentration of the cellulose ethers used in sprayable paints $(<1 \%)$ is usually lower than the $2 \%$ concentration examined here. However as the lower concentration EHEC and hmEHEC solutions have viscosity $\eta<100 \mathrm{mPa}$ s, the time required to initially establish the stretched liquid bridge in the CaBER device $(\sim 50 \mathrm{~ms})$ is longer than that required for the capillary-driven pinch-off. Rodd et al. ${ }^{77}$ examined the practical limits of the capillary break-up rheometer (with a CaBER device) and note that the device cannot be used for weakly elastic (De $=1$ or $\lambda<1 \mathrm{~ms}$ ) or low viscosity fluids $(\mathrm{Oh}<0.1)$. For the range of cellulose concentrations studied $(0.2<c<2.4 \%)$, only solutions with $c>2 \%$ are sufficiently viscous to sustain a viscocapillary balance. Shear thinning further reduces the effective viscosity of the solutions implying that capillary break-up of typical water-borne cellulose solutions occurs in a low Oh regime, where elastocapillary effects are hard to observe on experimentally accessible length-scales.
However, it is well-established that the response of highly elastic fluids can be measured for lower viscosity fluids (Ohnesorge number Oh $<0.1$ ), ${ }^{77}$ if the Deborah number, De $\mathrm{O}(1)$. For a dilute polymer solution, the relaxation time can be estimated using Zimm's theory, using the following expression:

$$
\lambda_{\mathrm{Z}}=F \frac{[\eta] M_{\mathrm{w}} \eta_{\mathrm{s}}}{N_{\mathrm{A}} k_{\mathrm{B}} T}
$$

where $M_{\mathrm{w}}$ is the polymer molecular weight, $N_{\mathrm{A}}$ is Avogadro's number, $k_{\mathrm{B}}$ is the Boltzmann constant, $T$ is the absolute temperature and $[\eta]$ is the intrinsic viscosity. The pre-factor can be approximated by the Riemann zeta function $F \simeq 1 / \zeta(3 v)=1 / \sum_{i=1}^{\infty}\left(1 / i^{3 v}\right)$ in which $v$ represents the solvent quality exponent, and it varies from $\nu=0.5$ for an ideal chain in a theta solvent to $\nu=0.588$ for a coil in a good solvent. ${ }^{128}$ Using $[\eta]=3.33 \mathrm{dL} \mathrm{g}^{-1}$ and $M_{\mathrm{w}}=240000$ Daltons (from our shear characterization), we get $\lambda_{\mathrm{z}}=0.014 \mathrm{~ms}$. The characteristic time for relaxation as determined in extensional experiments using techniques like CaBER is typically found to be concentrationdependent even for solutions considered to be dilute i.e. $c<$ $c^{*} .^{18,69,129}$ The overlap concentration for the EHEC dispersions is estimated using $c^{*} \approx 1 /[\eta]$ to be $c=0.3 \%$ by weight (where $c$ in weight percent is equivalent to the absolute value in units of $\mathrm{g} \mathrm{dL}^{-1}$ ). Above the overlap concentration, the relaxation time for flexible coils in an unentangled semi-dilute solution in a good solvent increases with concentration, $c$ or volume fraction $\phi$ (ref. 128) as

$$
\lambda_{\mathrm{SD}} \approx \frac{\eta_{\mathrm{s}} b_{\mathrm{K}}^{3}}{k_{\mathrm{B}} T} N_{\mathrm{K}}^{2} \phi^{(2-3 v) /(3 v-1)}
$$

here $b_{\mathrm{K}}$ is the Kuhn length and $N_{\mathrm{K}}$ is the number of Kuhn segments per chain, and as mentioned previously the volume fraction $\phi$ is related to the mass concentration $c^{\prime}$ by the expression $\phi=c^{\prime} N_{\mathrm{K}} b_{\mathrm{K}}{ }^{3} N_{\mathrm{A}} / M_{\mathrm{w}}$. Using Zimm's formula for good solvents, we can rewrite the above expression as $\lambda_{\mathrm{SD}} \approx$ $\lambda_{\mathrm{Z}} N_{\mathrm{K}}{ }^{2-3 v} \phi^{(2-3 v) /(3 v-1)}$, and using $v=0.588$, the estimate simplifies to be $\lambda_{\mathrm{SD}} \approx \lambda_{\mathrm{Z}} N_{\mathrm{K}}{ }^{0.33} \phi^{0.31}$. At the overlap concentration, $\phi=\phi^{*} \approx 1 / N_{\mathrm{K}}{ }^{3 v-1}$, and therefore $\lambda_{\mathrm{SD}} / \lambda_{\mathrm{Z}} \approx\left(\phi / \phi^{*}\right)^{0.31}$ or equivalently, $\lambda_{\mathrm{SD}} / \lambda_{\mathrm{Z}} \approx\left(c / c^{*}\right)^{0.31}$. Thus even for a $2 \mathrm{wt} \%$ solution of EHEC (with $c / c^{*} \approx 7$ ), the estimated relaxation time $\lambda_{\mathrm{SD}} \approx$ $0.025 \mathrm{~ms}$ of the EHEC solution is much less than a millisecond for these solutions, and it is difficult to establish an elastocapillary balance in such a weakly elastic fluid.

Only a few studies have probed the extensional response of dilute and semi-dilute polysaccharide solutions using CaBER. In contrast to the aqueous cellulose ether dispersions examined here, either higher molecular weights or more viscous solvents were used to make measurements possible. In capillary breakup studies of semi-dilute dispersions made with methylhydroxyethyl cellulose (MHEC), Plog et al. ${ }^{130}$ used aqueous $\mathrm{NaOH}$ solutions to suppress associations citing experimental difficulties with pure aqueous solutions. In their study, the MHEC solutions in aqueous $\mathrm{NaOH}$ contained a cellulose backbone with a higher degree of polymerization and higher intrinsic viscosity $\left(3.56-17.38 \mathrm{dL} \mathrm{g}^{-1}\right.$ compared to $3.33 \mathrm{dL} \mathrm{g}^{-1}$ for 
hmEHEC), and therefore $2 \%$ by weight solutions provided a measurable extensional viscosity and relaxation time. We have also probed the capillary break-up of cellulose $(\mathrm{DP}=800)$ in an ionic liquid - 1-ethyl-3-methylimidazolium acetate (EMIAc) - for which the solvent viscosity was $\eta_{\mathrm{s}}=0.1 \mathrm{~Pa}$ s (i.e. 100 times higher than the viscosity of water), and found relaxation times in the range $30-1700 \mathrm{~ms}$ for concentrations in the range $0.5-8 \%$ by weight. ${ }^{16}$ Since the relaxation time scales with the solvent viscosity, a simple scaling estimate for an aqueous solution of any cellulose ether with similar DP would give a relaxation time of $0.6 \mathrm{~ms}$ for a $2 \%$ solution. Duxenneuner et al. ${ }^{113}$ reported CaBER measurements for another polysaccharide, hydroxypropyl ether guar gum (HPEGG) in aqueous solutions with concentrations as low as $c=0.17 c^{*}$. Again the much higher molecular weight $\left(M_{\mathrm{w}}=2.3 \times 10^{6}\right.$ Daltons $)$ and a higher intrinsic viscosity of $13.2 \mathrm{dL} \mathrm{g}^{-1}$ for the HPEGG dispersions facilitated CaBER measurements of the extensional relaxational time, with relaxation times $\lambda>1 \mathrm{~ms}$ and $\operatorname{Tr}>10$ for both dilute and semi-dilute dispersions.

Like the cellulose ethers studied here, many of the complex fluids that are used in spraying applications or in inkjet printing are only very weakly elastic, i.e. the relaxation times of such fluids are small (typically significantly less than $1 \mathrm{~ms}$ ), and most sprayable or jettable fluids possess viscosities less than $100 \mathrm{mPa}$ s, which makes extensional rheology measurements with the CaBER instrument very difficult. Furthermore, the extension rates encountered in a real processing application can be as large as $10^{6} \mathrm{~s}^{-1}$ and are much higher than the values that can be established in a capillary thinning experiment, where the maximum extension rate resolved in elasto-capillary thinning is limited $^{77}$ to around $1000 \mathrm{~s}^{-1}$. Therefore, we turn to detailed studies of capillary-driven thinning and break-up during jetting as a means of observing pinch-off dynamics for low viscosity and weakly elastic fluids, as described in the next section.

\subsection{Jetting and break-up of associative cellulose ethers}

A representative set of images showing instability growth and pinch-off dynamics for jets of a $0.23 \%$ hmEHEC solution as a function of perturbation frequency (proportional to dimensionless wavenumber, $k R_{0}$ ) are shown in Fig. 10 and 11 . The images incorporate detailed information about the nature of viscoelastic jet break-up (symmetric at high wavenumbers, $k$ becoming progressively more asymmetric at lower wavenumbers) and the formation of satellite drops (Movies M1-M3 added as ESI $\dagger$ showing $k R_{0}=0.53,0.64$ and 0.78).

The dotted line shown in Fig. 10 superimposes the dispersion curve for an inviscid fluid (computed using eqn (3)). A particularly simple and symmetric mode of break-up is observed for frequencies above the critical frequency, $f^{*}$, at which the shortest breakup length is measured. In this regime, jet break-up can be used to extract extensional viscosity information. ${ }^{70,71}$ The critical frequency corresponds to the wavenumber, $k_{\max } R_{0}=0.69$ for an inviscid jet, and the peak shifts to a slightly lower value for low viscosity, weakly viscoelastic jets. ${ }^{\mathbf{1 1 0 2}}$ At lower perturbation frequencies (longer wavelength modes), additional higher frequency modes with higher growth rates compete with the primary excitation mode that corresponds to the applied frequency. Computational analysis shows that these higher frequency modes lead to the formation of finer scale features such as satellite drops. However, if the excitation frequency is higher than the critical frequency, so that $f>f^{*}$ the shorter wavelength modes that correspond to the development of asymmetries now grow more slowly than the primary varicose mode and are thus suppressed. In Fig. 11 we show a sequence of images of the temporal evolution of the capillary thinning process observed during jetting. In contrast to low viscosity, Newtonian fluid jets (at the same We and Oh), the jets of the cellulose ether solutions examined in this study, show no satellite drop formation. Thus it is clear that nonlinear effects due to tensile stresses generated by the deformation of polymer chains in response to the elongational flow within the neck delay pinch-off sufficiently to suppress satellite formation.

The spatiotemporal evolution of a viscoelastic jet can be described in terms of the jet radius $R(z, t)$ where $z$ represents the distance from the nozzle and $t$ is the time. A Lagrangian observer " $P$ " located on the jet moving with velocity $V_{\mathrm{j}}$ reports the change in radius as $R=R_{\mathrm{P}}\left(z=V_{\mathrm{j}} t\right)$. From the sequence of images in Fig. 11, the progressive decrease in the radius of the neck $R=R_{\mathrm{P}}\left(z=V_{\mathrm{j}} t\right)$ is extracted for an excitation with $k R_{0}=0.78$ and the data are shown, plotted as a function of dimensionless time in Fig. 12. The corresponding extensional rates computed using eqn (6) are also shown. We note that the Rayleigh time used for non-dimensionalizing the abscissa is $t_{\mathrm{R}}=0.11 \mathrm{~ms}$, and thus the whole capillary pinch-off event is effectively complete in a time of $1.8 \mathrm{~ms}$. This is much faster than the typical time required in our CaBER experiment for establishing a stretched liquid bridge. Though Schümmer and Tebel ${ }^{78}$ were probably the first to motivate the use of a jetting-based elongational rheometer, their own data show large variability with perturbation amplitude and imposed frequency (which was varied using a speaker) and jet velocity. By identifying the significant role of perturbation wavenumbers in dictating the kinematics of capillary-driven thinning and break-up during jetting, and by limiting ourselves to forcing frequencies in the range $f>f^{*}$ for analysis, we were able to create repeatable measurements of the transient extensional viscosity and relaxation time, and we obtained similar values with different perturbation frequencies, jet velocities and perturbation amplitudes. By using high driving frequencies and jet velocity, we can apply extensional deformations at rates that are higher than the estimated Zimm relaxation rate of the chain, and also faster than the disassociation rate of stickers present on the hmEHEC chains. We note that in Fig. 12 the neck radius decreases linearly with time close to break-up. In the previous section, we noted that a linear decrease of radius with time is characteristic of the viscocapillary response of Newtonian fluids. ${ }^{125}$ If we use eqn (12) and the measured slope (0.09) of the curve shown in Fig. 12, we obtain an effective viscosity $\eta_{\text {eff }}=22 \eta_{0}$ that is substantially larger than the value of the zero shear viscosity, $\eta_{0}$ value measured for $0.23 \%$ hmEHEC solution. The apparent Ohnesorge number $\mathrm{Oh}=0.55$ is computed to be 22 times higher than the value based on zero shear viscosity, Oh $=0.025$. Unlike the CaBER observations with the $2 \%$ cellulose ether solutions that 


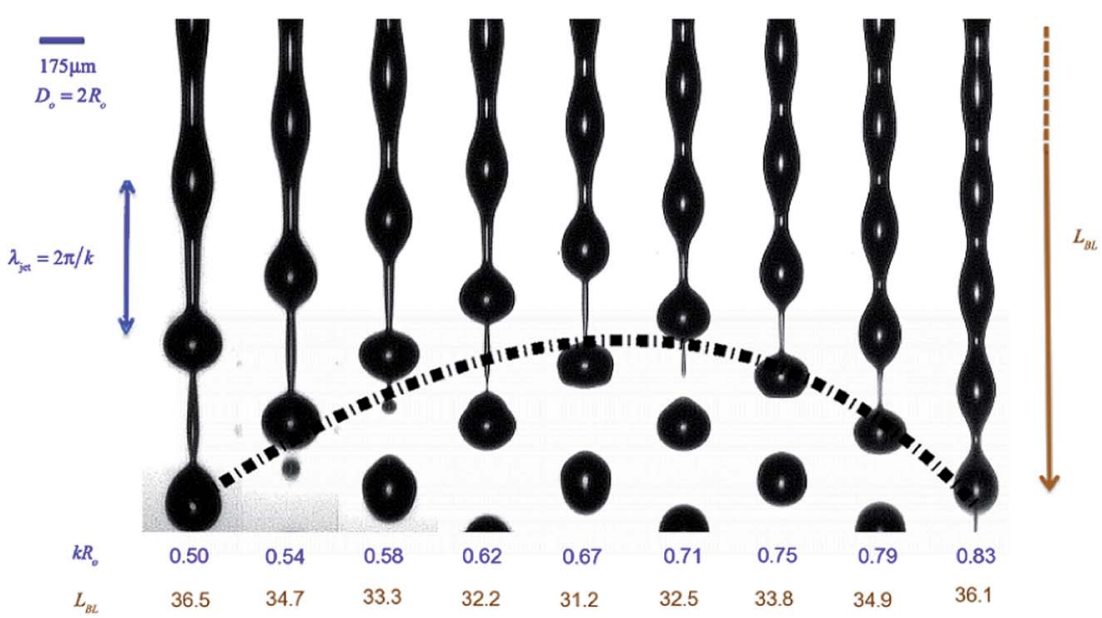

Fig. 10 Forced jetting of $0.23 \% \mathrm{hmEHEC}$ solutions. The imposed perturbation frequency $\left(k R_{0}\right)$ is varied using an annular piezoelectric transducer. The black, dotted line shows the break-up length expected on the basis of linear stability analysis for inviscid fluid. The jet is backlit, and it refracts light away from the image plane causing the jet and drops to appear dark, except at the very center where light passes straight through the jet towards the camera.

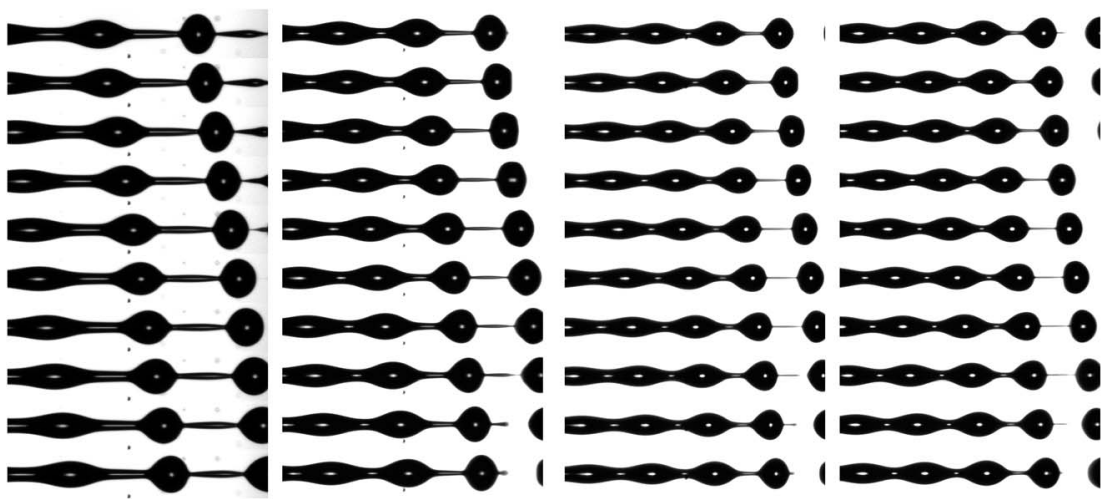

Fig. 11 Jet breakup of $0.225 \% \mathrm{hmEHEC}$. The forcing frequency in each column is $k R_{0}=0.52(3.1 \mathrm{kHz}), k R_{0}=0.67(4.05 \mathrm{kHz}), k R_{0}=0.75(4.5 \mathrm{kHz})$ and $k R_{0}=0.82(4.90 \mathrm{kHz})$ respectively, and $\Delta f=0.1 \mathrm{~Hz}$. Each panel has 10 images, each separated by $20 \mu \mathrm{s}$. Note here $\mathrm{Oh}=0.025, \mathrm{We}=40$, and $\mathrm{Re}=160$

yielded a lower apparent Oh than computed on the basis of zero-shear viscosity, the value of Oh measured during jetting is substantially larger, and clearly, the presence of the hmEHEC chains has modified the final stage of the pinch-off dynamics.

In contrast to the Oldroyd-B model in which only an elastocapillary regime with exponentially slow thinning is predicted, nonlinear models that include anisotropy in the drag acting on polymer (e.g. the Giesekus model) or finitely extensible dumbbell models (such as the FENE-P model) allow a finite time break-up accompanied by a linear decrease in radius close to the pinch-off time i.e. the minimum radius in the neck should scale as $R(t) / R_{0} \approx B\left(t_{\mathrm{c}}-t\right) \cdot{ }^{\mathbf{9 , 1 3 1 , 1 3 2}}$ In the FENE-P model, the polymer chains reach a fully stretched state during elastocapillary thinning and behave as a dilute suspension of rigid rods that increase the total effective viscosity of the fluid. ${ }^{132,133}$ Entov and Hinch ${ }^{\mathbf{1 0 4}}$ used the FENE model to predict a linear decrease in radius with time of the form given above with $B=\sigma / 6 \eta_{\text {eff }} R_{0}$ where $\eta_{\text {eff }}$ is the effective viscosity of the fully stretched dumbbells. Renardy, and Fontelos and Li argued that the prefactor
$B$ is dependent on the constitutive model used for describing finite extensibility effects and the conformation-induced change in the magnitude of drag coefficient.9,131,134,135 The evolution and break-up of jets of viscoelastic fluids of Giesekus and FENE-P type both predict a self-similar and linear decrease in neck diameter close to pinch-off, quite similar to the dynamics observed in Fig. 11c and $\mathrm{d}$ and 12. The fore-aft symmetry of the thin thread or ligament connecting the large primary drops indicates that fluid inertia is not important during the break-up process.

The extensional flow induced stretching of polymer chains results in a linear decrease in radius of the thinning ligament with time which can be written in the following dimensionless form:

$$
\frac{R(t)}{R_{0}} \approx \frac{\sigma}{6 \eta_{\mathrm{eff}} R_{0}}\left(t_{\mathrm{c}}-t\right)=p\left(\frac{\mathrm{Oh}^{-1}}{\operatorname{Tr}_{\infty}}\right)\left(\frac{t_{\mathrm{c}}-t}{t_{\mathrm{R}}}\right)
$$

Fitting the linear decrease in radius with time observed in Fig. 12 with eqn (15) gives a slope of $0.05\left(\operatorname{Tr}_{\infty} \mathrm{Oh}\right)^{-1}=-0.09$, 


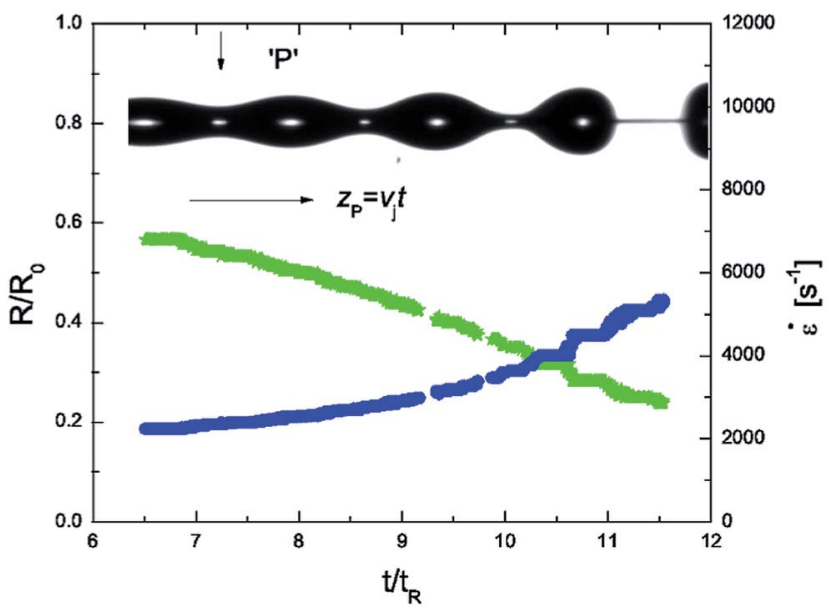

Fig. 12 Jetting analysis of $0.23 \%$ hmEHEC solution showing scaled radius $R(t) / R_{0}$ Vs. scaled time for Langragian point ' $P$ ' on the thinning neck (green symbol, left axis). The forcing frequency is $k R_{0}=0.78$. The blue circles on the right hand axis show the extension rate (see eqn (6)) as a function of scaled time.

and consequently the limiting value of Trouton ratio obtained for fully stretched chains is $\operatorname{Tr}_{\infty}=22$. Irrespective of the details of the model used, the observation of such a linear scaling implies that the limiting value of Trouton ratio, $\operatorname{Tr}_{\infty}$, associated with a high strain, and high extension rate (i.e. large Wi) flow is being measured in this experiment. Due to the enhanced resistance to pinch-off, the cylindrical ligament remains slender for a longer time than the value expected from the viscocapillary scaling based on the zero shear viscosity. The enhanced viscoelastic resistance to pinch-off also leads to the absence of satellite drops at different perturbation frequencies, as shown in Fig. 11 and 12.

In recent work using drop on demand inkjet printing with weakly elastic fluids, ${ }^{72,73,136}$ it has been observed that the macromolecules approach their maximum elongation in the thinning neck between drops. Macromolecules reach a high degree of extension in a transient flow when two conditions: ${ }^{5,90}$ 1) a critical rate condition and 2) the accumulated strain condition are satisfied. The first condition requires the velocity gradient (or deformation rate), $\dot{\varepsilon}$, to exceed the relaxation rate of the macromolecules, $1 / \lambda$. For dilute solutions, the simplest estimate of this rate is based on $\dot{\varepsilon}>\lambda_{\mathrm{z}}$ or Zimm relaxation time, ${ }^{137}$ which is the longest relaxation time of the molecule in its coiled state. The accumulated strain condition requires a large strain rate to be maintained for long enough for large molecular strains to develop (flexible high molecular weight polymers can require stretching by 100 -fold to become fully stretched). According to the relaxation times given above and the magnitudes of the measured extension rates shown in Fig. 12 and 13, condition (1) is satisfied in the jetting experiments. The experimental results indicate that the observed pinch-off dynamics are characteristic of fully stretched and oriented chains, and the maximum observable fluid strain in the current set-up is estimated using eqn (7) to be $\varepsilon_{\mathrm{H}} \approx 7$. To determine whether the cellulose ethers are likely to become fully stretched in these jetting experiments, we need to estimate the extensibility of the polymer chains.
For the Kuhn chain, the maximum extension or finite extensibility limit of a chain can be estimated by

$$
L \equiv \frac{R_{\max }}{\left\langle R_{0}^{2}\right\rangle^{1 / 2}}=\frac{N_{\mathrm{K}}^{2 v} b_{\mathrm{K}}^{2}}{N_{\mathrm{K}} b_{\mathrm{K}}}=N_{\mathrm{K}}^{1-\nu}
$$

here the mean square end-to-end distance $\left\langle R_{0}{ }^{2}\right\rangle$ gives an estimate for coil size and the contour length of the chain. $R_{\max }$ gives the maximum size to which a chain can be stretched. We can use our measured value of $[\eta]=3.88 \mathrm{dL} \mathrm{g}^{-1}$ for the EHEC solution to obtain an estimate of the root-mean-squared end-toend length at equilibrium, $\left\langle R_{0}{ }^{2}\right\rangle^{1 / 2}$ using the formula

$$
\left\langle R_{0}^{2}\right\rangle=\left([\eta] M_{\mathrm{w}} / \Phi_{\infty}\right)^{2 / 3}
$$

where $M_{\mathrm{w}}$ is the molecular weight of the cellulose ether $\left(M_{\mathrm{w}} \approx\right.$ $240 \mathrm{kDa}$ for EHEC of DP $=800$ ) and $\Phi_{\infty}=2.1 \times 10^{23} \mathrm{~mol}^{-1}$ is the Fox-Flory constant for a good solvent. ${ }^{\mathbf{1 3 8 , 1 3 9}}$ We obtain a value of $\left\langle{R_{0}}^{2}\right\rangle^{1 / 2} \approx 75 \mathrm{~nm}$. We can estimate $R_{\max } \approx 412 \mathrm{~nm}$ for the contour length of the DP $=800$ cellulose ether molecule, by taking the length of a single AGU repeat unit to be $l_{\mathrm{AGU}}=$ $0.515 \mathrm{~nm}$ (ref. 12 and 140) (for detailed computation, see the work by Tsvetkov ${ }^{141}$ ). From eqn (16), this gives ${N_{\mathrm{K}}}^{1-\nu}=5.5$ and since water is a good solvent for EHEC, ${ }^{\mathbf{1 4 2}}$ we use the exponent $v=0.588$, to get $N_{\mathrm{K}}=62$ and $b_{\mathrm{K}} \approx 6.6 \mathrm{~nm}$, or $K \approx 12 l_{\mathrm{AGU}}$. Most importantly, the ratio of contour length to root-mean-squared end-to-end length is very modest, $L=R_{\max } /\left\langle R_{0}{ }^{2}\right\rangle^{1 / 2} \approx 5.5$, suggesting that only moderate strains are required to completely extend this semi-flexible macromolecule.

To further test the validity of these arguments, we carried out additional jetting-based rheometry studies on a dilute polyethylene oxide (PEO) solution $\left(M_{\mathrm{w}}=300000\right.$ Daltons) dissolved in a glycerol-water (46:54) mixture (viscosity matched with
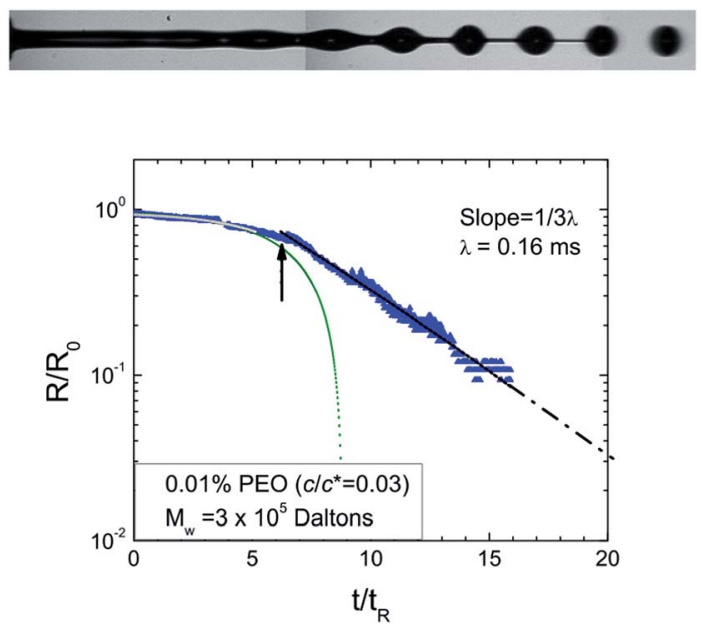

Fig. 13 Jetting analysis for $0.01 \%$ PEO solution $\left(M_{w}=300000\right.$ Daltons) in glycerol-water (46/54 wt\%) with $\mathrm{Oh}=0.05, \mathrm{We}=20, t_{\mathrm{R}}=$ $0.11 \mathrm{~ms}$, and $\mathrm{De}=0.5$ showing evolution in the scaled neck radius vs. scaled time. The forcing frequency is $k R_{0}=0.78$. The dotted green line shows that initially the thinning dynamics follow the expected inertiocapillary scaling, and the black line (dash-dotted) shows that beyond the point denoted by arrow, the elastocapillary scaling (see eqn (10)) is observed. 
cellulose ether solution). The molecular weight of the PEO is chosen to be comparable to the cellulose ethers, and the concentration chosen, $c=0.01 \%$, corresponds to an extremely dilute solution $(c[\eta] \approx 0.03)$. Because of the flexibility of PEO chains, we obtain $N_{\mathrm{K}}=3750$, in contrast to $N_{\mathrm{K}}=62$ for the cellulose ethers. The corresponding extensibility for PEO chains is $L \approx 27$, which is nearly five times larger than the extensibility of the cellulose ethers. The capillary-driven thinning behavior exhibited by this dilute viscoelastic solution is shown in Fig. 13 . The more flexible, extensible PEO solution displays exponential thinning dynamics, a signature feature of the elastocapillary scaling discussed previously (see eqn (10)). For PEO solutions, a relaxation time of $\lambda=0.16 \mathrm{~ms}=3 \lambda_{\mathrm{z}}$, and a Trouton ratio as high as $\operatorname{Tr}=300$ can be computed. In the context of spraying applications, it is clearly an advantage to have cellulose-based thickeners or rheology modifiers as their limited extensibility ensures that extensional viscosity effects are bounded.

Analysis of capillary break-up during jetting of 0.4\% EHEC and hmEHEC solutions shown in Fig. 14 also shows a qualitatively similar response to the $0.23 \%$ hmEHEC solutions. The radius versus time data are shown along with the corresponding extension rate for both the solutions. Using eqn (15), we can again estimate limiting Trouton ratios of $\operatorname{Tr}_{\infty}=10$ for $0.4 \%$ EHEC solution and $\operatorname{Tr}_{\infty}=8$ for hmEHEC solutions. The $0.4 \%$ EHEC and hmEHEC solutions can be considered semi-dilute, but since the intrinsic viscosity of these solutions is $3.88 \mathrm{dL}$ $\mathrm{g}^{-1}$ and $3.33 \mathrm{dL} \mathrm{g}^{-1}$ respectively, the effect of stickers is hardly visible in the extensional response of the hmEHEC solutions.

An estimate for the upper bound of the extensional viscosity or Trouton ratio for fully extended EHEC chains can be made by assuming that the fully extended chains behave like rods of length $R_{\max }$. The estimated value of the Trouton ratio $\operatorname{Tr}_{\infty}^{\mathrm{B}}$ computed using Batchelor's theory for rods ${ }^{\mathbf{8 8 , 1 4 3}}$ gives:

$$
\operatorname{Tr}_{\infty}^{\mathrm{B}}=\frac{\pi}{3} \frac{\eta_{\mathrm{s}}}{\eta_{0}} \frac{c^{\prime} N_{\mathrm{A}} R_{\max }{ }^{3}}{M_{\mathrm{w}} \ln (\pi / \phi)}=\frac{\pi}{3} \frac{\eta_{\mathrm{s}}}{\eta_{0}} \frac{\phi N_{\mathrm{K}}{ }^{2}}{\ln (\pi / \phi)}
$$

where all variables are the same as before and the volume fraction $\phi$ is related to the concentration $c^{\prime}$ in mass/volume by the expression $\phi=c^{\prime} N_{\mathrm{K}} b_{\mathrm{K}}^{3} N_{\mathrm{A}} / M_{\mathrm{w}}$. The estimated value of $\operatorname{Tr}_{\infty}^{\mathrm{B}} \approx$ 111 using eqn (18) is much higher than the measured value, and suggests that the semi-flexible EHEC chains are not fully aligned. For finitely extensible polymer molecules with extensibility $L$, the rate-dependent FENE-P model gives an alternative (and better) estimate for the steady extensional viscosity value at close to full extension:

$$
\eta_{\mathrm{E} \infty}^{\mathrm{FP}}=3 \eta_{\mathrm{s}}+2 \eta_{\mathrm{P}} L^{2}
$$

For the cellulose ether chains at $c^{*}$, using the appropriate values of extensibility, $L=5.5$ and the measured polymer contribution to shear viscosity, $\eta_{\mathrm{P}}=0.7 \mathrm{mPa} \mathrm{s}$ we find the expected maximum value of the Trouton ratio to be $\operatorname{Tr}_{\infty}^{\mathrm{FP}} \approx 64$. The experimental value of $\operatorname{Tr}_{\infty} \approx 22$ for $0.23 \%$ HMEHEC measured using jet rheometry is thus reasonably close to the estimate given that the polymer samples used in our study are polydisperse.
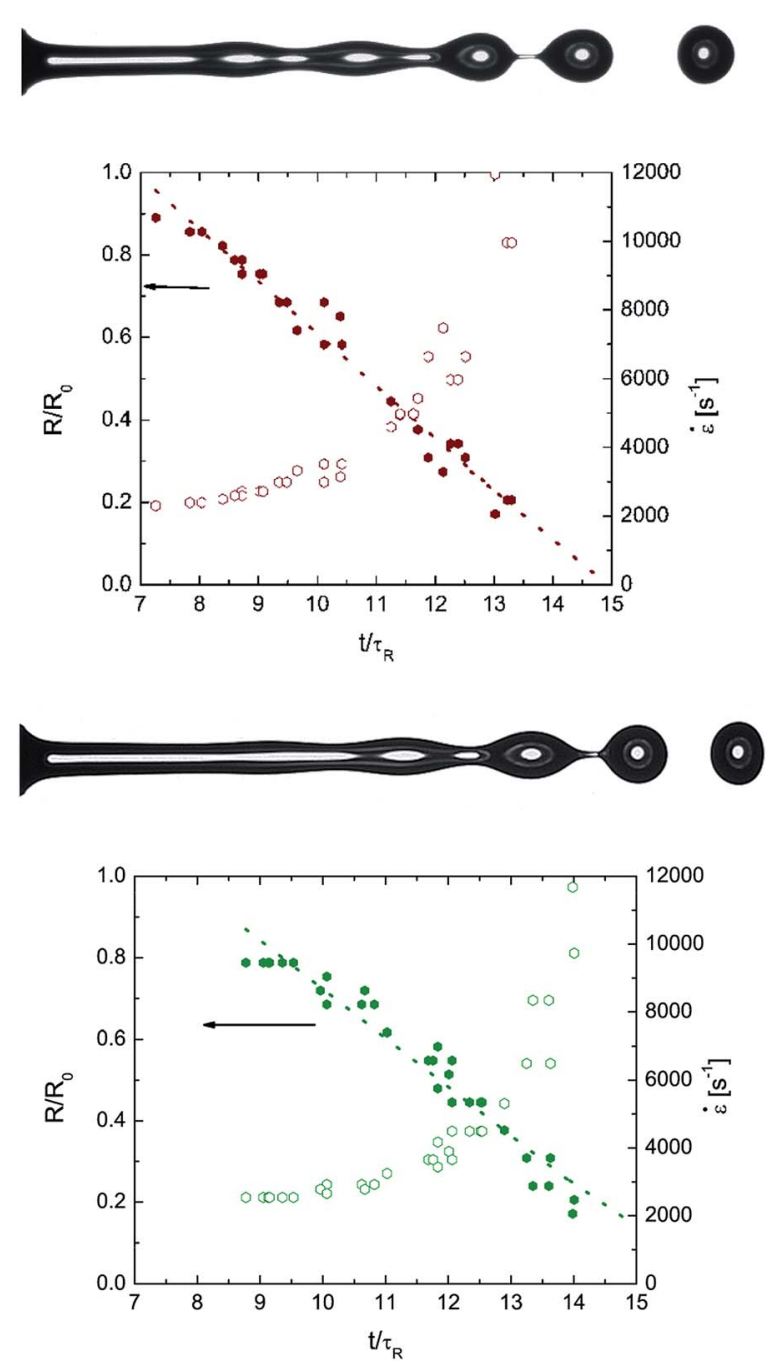

Fig. 14 Neck radius vs. time comparison of $0.4 \%$ EHEC \& $0.4 \%$ hmEHEC solutions, obtained by analyzing ROJER data. The data are based on images acquired using the JetXpert system.

\section{Conclusions}

The rheological response of EHEC and hmEHEC solutions to a range of extensional deformations was studied by using excess pressure drop and flow induced birefringence measurements during steady planar extensional flow in a microfluidic crossslot device and by a recently developed technique referred to as Rayleigh Ohnesorge Jetting Extensional Rheometry or ROJER that follows the capillary thinning and break-up of thin fluid ligaments that form between drops during jetting of weakly viscoelastic fluids. The reduction of the characteristic geometric dimensions down to the microscale in both the ROJER and cross-slot instruments, together with the use of electro-optic imaging techniques, enables accurate measurements of the extensional viscosities of these polymer solutions using relatively small scales, and allows access to the high extension rates that are of most relevance to applications such as paint spraying and coating operations. The ROJER technique can be used with opaque fluids and access comparatively higher extensional rates 
than cross-slot extensional rheometry, which requires optically transmissive fluids. Both techniques are ideal for use with other polysaccharide-based biological samples and industrial fluids, as the evaporation of volatile solvents is negligible and temperature control can be implemented relatively simply. We hope that the techniques and methodology described here will find widespread use in formulating and studying specialized inks for electronic, photovoltaic and biomedical print-based manufacturing technologies. ${ }^{47,48,144}$

Quantitative comparison between the extensional rheology of EHEC solutions and the hydrophobically modified analogue, hmEHEC, shows that the presence of additional C-14 hydrophobic groups as stickers provides the hmEHEC dispersions with a much larger extensional viscosity at low extension rates. The extensional viscosity and flow-induced birefringence of the semiflexible EHEC solutions increase monotonically with extensional rate and with concentration. By contrast, the hmEHEC solutions exhibit extensional thinning behavior and a reduction in the specific birefringence at higher concentrations, indicative of inter-chain effects. The dramatic extensional thinning behavior at high extension rates shown by hmEHEC dispersions is quite reminiscent of their response to high shear rates. At the highest shear and extensional rates, the physical associations between the chains are no longer effective and the hydrophobically modified cellulose ethers display low viscosities, which make hmEHEC dispersions ideal for use in jetting and spraying applications. In the jetting experiments, analysis of the pinch-off dynamics exhibited by the cellulose ether solutions reveals a linear decay of neck radius with time, which is a scaling characteristic of finite extensibility. The EHEC and hmEHEC solutions display quite moderate values of the limiting Trouton ratio (typically no more than $\operatorname{Tr}_{\infty} \approx 20$ ), and the experimental values are in agreement with estimates based on finite extensible nonlinear elastic (FENE-P) models. Again this bounded increase in the extensional viscosity is favorable for spraying and coating operations as it eliminates the stringiness and beads-on-strings structures that plague many other systems such as dilute solutions of flexible long chain macromolecules. ${ }^{136,145,146}$

Of course, the jetting or spraying behavior of a complex multicomponent fluid such as paint is influenced not only by the polymer viscoelasticity and extensibility, but also by the combined and coupled effects of colloidal particles, surfactants and other additives that are present in the dispersion. ${ }^{32,33,136}$ However the present study illustrates that the flow-induced destruction of the transient network that is initially established in solutions of associative polymer thickeners can help produce the requisite low viscosities required for drop formation in jetting or spraying. The creation of a physical network at low deformation rates provides the dispersions with the high viscosity and large gel strength needed to stick to surfaces and confers such paints and coatings with desirable leveling and sagging properties. In spite of the extensional thinning effects, the cellulose ether dispersions still present a greater extensional resistance to pinch-off than corresponding Newtonian fluids with similar shear viscosities, and this delays the final pinch-off event substantially, therefore reducing the size of satellite drops (if present at all) below experimental resolution. Tailoring the number of stickers and the strength of the association in the transient junctions and controlling the distance between hydrophobic stickers along the hydrophilic backbone each provide possible mechanisms for the design of a stable and sprayable multicomponent complex fluid. In each case, an understanding of the rate-dependent response of the fluid to the very large imposed shear and extensional deformation rates characteristic of coating operations is essential. The different extensional rheometer configurations presented in this paper show that both the transient and steady extensional rheological response of weakly viscoelastic fluids can now accurately be measured at deformation rates up to $\dot{\varepsilon} \approx 10^{4} \mathrm{~s}^{-1}$.

\section{Acknowledgements}

VS designed the experiments, analyzed the data and wrote the paper in collaboration with and under supervision of GHM and PTH. SJH designed and performed the cross-slot rheometry and contributed to the related sections of the manuscript. VS and JS designed the ROJER set-up. AkzoNobel provided the project funding. AS supplied the cellulose ethers and, together with PTH, provided overall project guidance as well as questions and insights into the application needs in the paint industry. The jetting images for the $0.4 \%$ cellulose ether were obtained using the JetXpert with the assistance of BK. SJH gratefully acknowledges financial support from NASA Microgravity Fluid Sciences (Code UG) grant NNX09AV99G, the European Commission under Marie Curie action FP7-PEOPLE-2011-IIF grant 298220 and the Okinawa Institute of Science and Technology Graduate University (OIST).

\section{References}

1 F. T. Trouton, Proc. R. Soc. London, Ser. A, 1906, 77, 426-440.

2 C. J. S. Petrie, Elongational Flows, Pitman, London, 1979.

3 C. J. S. Petrie, J. Non-Newtonian Fluid Mech., 2006, 137, 1523.

4 C. W. Macosko, Rheology: Principles, Measurements and Applications, VCH Publishers Inc, New York, 1994.

5 S. J. Haward, V. Sharma and J. A. Odell, Soft Matter, 2011, 7, 9908-9921.

6 G. Juarez and P. E. Arratia, Soft Matter, 2011, 7, 9444-9452.

7 D. J. Mai, C. Brockman and C. M. Schroeder, Soft Matter, 2012, 8, 10560-10572.

8 A. L. Yarin, Free Liquid Jets and Films: Hydrodynamics and Rheology, Longman Scientific \& Technical, 1993.

9 G. H. McKinley, Rheol. Rev., 2005, 1-48.

10 E. Tekin, P. J. Smith and U. S. Schubert, Soft Matter, 2008, 4, 703-713.

11 G. Bhatara, E. S. G. Shaqfeh and B. Khomami, J. Rheol., 2005, 49, 929-962.

12 R. Lapasin and S. Pricl, Rheology of Industrial Polysaccharides: Theory and Applications, Chapman \& Hall, London, 1995.

13 Polysaccharides: Structural Diversity and Functional Versality, ed. S. Dumitriu, Marcel Dekker, New York, 2nd edn, 2005. 
14 B. Nyström, A.-L. Kjøniksen, N. Beheshti, K. Zhu and K. D. Knudsen, Soft Matter, 2009, 5, 1328-1339.

15 L. Karlson, F. Joabsson and K. Thuresson, Carbohydr. Polym., 2000, 41, 25-35.

16 S. J. Haward, V. Sharma, C. P. Butts, G. H. McKinley and S. S. Rahatekar, Biomacromolecules, 2012, 13, 1688.

17 J. P. Tatham, S. Carrington, J. A. Odell, A. C. Gamboa, A. J. Muller and A. E. Saez, J. Rheol., 1995, 39, 961-986.

18 D. A. Z. Wever, F. Picchioni and A. A. Broekhuis, Prog. Polym. Sci., 2011, 36, 1558-1628.

19 E. Puchelle, J. Zahm and C. Duvivier, Biorheology, 1983, 20, 239.

20 P. Erni, M. Varagnat, C. Clasen, J. Crest and G. H. McKinley, Soft Matter, 2011, 7, 10889-10898.

21 M. S. Green and A. V. Tobolsky, J. Chem. Phys., 1946, 14, 8092.

22 A. Tripathi, K. C. Tam and G. H. McKinley, Macromolecules, 2006, 39, 1981-1999.

23 T. Annable, R. Buscall, R. Ettelaie and D. Whittlestone, J. Rheol., 1993, 37, 695-726.

24 M. A. Winnik and A. Yekta, Curr. Opin. Colloid Interface Sci., 1997, 2, 424-436.

25 F. Tanaka, Polymer Physics: Applications to Molecular Association and Thermoreversible Gelation, Cambridge Univ Press, 2011.

26 R. R. Eley, Rheol. Rev., 2005, 173-240.

27 M. Rubinstein and A. N. Semenov, Macromolecules, 1998, 31, 1386-1397.

28 M. Rubinstein and A. N. Semenov, Macromolecules, 2001, 34, 1058-1068.

29 Paint and Surface Coatings: Theory and Practice, ed. R. Lambourne and T. A. Striven, Woodhead Publishing Ltd, Cambridge, U. K., 2nd edn, 1999.

30 S. Paul, Surface Coatings: Scienceand Technology, John Wiley \& Sons, New York, 1985.

31 O. A. Basaran, H. Gao and P. P. Bhat, Annu. Rev. Fluid Mech., 2013, 45, 85-113.

32 J. Eggers, Rev. Mod. Phys., 1997, 69, 865-929.

33 J. Eggers and E. Villermaux, Rep. Prog. Phys., 2008, 71, 036601.

34 H. A. Stone, Annu. Rev. Fluid Mech., 1994, 26, 65-102.

35 C. Clasen, J. Eggers, M. A. Fontelos, J. Li and G. H. McKinley, J. Fluid Mech., 2006, 556, 283-308.

36 J. Li and M. A. Fontelos, Phys. Fluids, 2003, 15, 922-937.

37 Y. Christanti and L. M. Walker, J. Non-Newtonian Fluid Mech., 2001, 100, 9-26.

38 Y. Christanti and L. M. Walker, J. Rheol., 2002, 46, 733-748.

39 R. P. Mun, J. A. Byars and D. V. Boger, J. Non-Newtonian Fluid Mech., 1998, 74, 285-297.

40 A. Bazilevsky, V. Entov and A. Rozhkov, in Third European Rheology Conference and Golden Jubilee Meeting of the British Society of Rheology, Elsevier, Edinburgh, UK, 1990, pp. 41-43.

41 Y. Amarouchene, D. Bonn, J. Meunier and H. Kellay, Phys. Rev. Lett., 2001, 86, 3558-3561.

42 S. L. Anna and G. H. McKinley, J. Rheol., 2001, 45, 115-138.
43 R. Sattler, S. Gier, J. Eggers and C. Wagner, Phys. Fluids, 2012, 24, 023101.

44 Y. Christanti and L. M. Walker, Atomization Sprays, 2006, 16, 777-790.

45 D. F. James and K. Walters, in Techniques of Rheological Measurement, ed. A. A. Collyer, Elsevier, New York, 1994, pp. 33-53.

46 T. Sridhar, J. Non-Newtonian Fluid Mech., 1990, 35, 85-92.

47 P. Calvert, Chem. Mater., 2001, 13, 3299-3305.

48 B. Derby, Annu. Rev. Mater. Res., 2010, 40, 395-414.

49 C. A. Wolden, J. Kurtin, J. B. Baxter, I. Repins, S. E. Shaheen, J. T. Torvik, A. A. Rockett, V. M. Fthenakis and E. S. Aydil, J. Vac. Sci. Technol., A, 2011, 29, 030801.

50 F. Galindo-Rosales, M. Alves and M. Oliveira, Microfluid. Nanofluid., 2013, 14, 1-19.

51 J. A. Odell and S. P. Carrington, J. Non-Newtonian Fluid Mech., 2006, 137, 110-120.

52 W. B. Zimmerman, J. M. Rees and T. J Craven, Microfluid. Nanofluid., 2006, 2, 481-492.

53 J. A. Pathak and S. D. Hudson, Macromolecules, 2006, 39, 8782-8792.

54 A. Keller and J. Odell, Colloid Polym. Sci., 1985, 263, 181201.

55 C. J. Pipe and G. H. McKinley, Mech. Res. Commun., 2009, 36, 110-120.

56 D. R. Link, S. L. Anna, D. A. Weitz and H. A. Stone, Phys. Rev. Lett., 2004, 92, 054503.

57 J. Soulages, M. S. N. Oliveira, P. C. Sousa, M. A. Alves and G. H. McKinley, J. Non-Newtonian Fluid Mech., 2009, 163, 9-24.

58 S. D. Hudson, F. R. Phelan, M. D. Handler, J. T. Cabral, K. B. Migler and E. J. Amis, Appl. Phys. Lett., 2004, 85, 335-337.

59 J. S. Lee, E. S. G. Shaqfeh and S. J. Muller, Phys. Rev. E: Stat., Nonlinear, Soft Matter Phys., 2007, 75, 040802.

60 J. S. Lee, R. Dylla-Spears, N. P. Teclemariam and S. J. Muller, Appl. Phys. Lett., 2007, 90, 074103.

61 J. Deschamps, V. Kantsler, E. Segre and V. Steinberg, Proc. Natl. Acad. Sci. U. S. A., 2009, 106, 11444-11447.

62 R. Dylla-Spears, J. E. Townsend, L. Jen-Jacobson, L. L. Sohn and S. J. Muller, Lab Chip, 2010, 10, 1543-1549.

63 P. A. Stone, S. D. Hudson, P. Dalhaimer, D. E. Discher, E. J. Amis and K. B. Migler, Macromolecules, 2006, 39, 7144-7148.

64 S. J. Haward, J. A. Odell, Z. Li and X.-F. Yuan, Rheol. Acta, 2010, 49, 633-645.

65 S. J. Haward, Rheol. Acta, 2010, 49, 1219-1225.

66 S. J. Haward, T. J. Ober, M. S. N. Oliveira, M. A. Alves and G. H. McKinley, Soft Matter, 2012, 8, 536-555.

67 P. E. Arratia, L. Cramer, J. P. Gollub and D. J. Durian, New J. Phys., 2009, 11, 115006.

68 P. E. Arratia, J. P. Gollub and D. J. Durian, Phys. Rev. E: Stat., Nonlinear, Soft Matter Phys., 2008, 77, 036309.

69 V. Tirtaatmadja, G. H. McKinley and J. J. Cooper-White, Phys. Fluids, 2006, 18, 043101.

70 V. Sharma, A. M. Ardekani and G. H. McKinley, in 5th Pacific Rim Conference on Rheology (PRCR-5), Sapporo, Japan, 2010. 
71 A. M. Ardekani, V. Sharma and G. H. McKinley, J. Fluid Mech., 2010, 665, 46-56.

72 D. C. Vadillo, W. Mathues and C. Clasen, Rheol. Acta, 2012, 51, 755-769.

73 D. C. Vadillo, T. R. Tuladhar, A. C. Mulji, S. Jung, S. D. Hoath and M. R. Mackley, J. Rheol., 2010, 54, 261.

74 L. Campo-Deano and C. Clasen, J. Non-Newtonian Fluid Mech., 2010, 165, 1688-1699.

75 G. H. McKinley and T. Sridhar, Annu. Rev. Fluid Mech., 2002, 34, 375-415.

76 M. Stelter and G. Brenn, J. Rheol., 2000, 44, 595-616.

77 L. E. Rodd, T. P. Scott, J. J. Cooper-White and G. H. McKinley, Appl. Rheol., 2005, 15, 12-27.

78 P. Schümmer and K. H. Tebel, Rheol. Acta, 1982, 21, 514516.

79 P. K. Bhattacharjee, D. A. Nguyen, G. H. McKinley and T. Sridhar, J. Rheol., 2003, 47, 269-290.

80 P. K. Bhattacharjee, J. P. Oberhauser, G. H. McKinley, L. G. Leal and T. Sridhar, Macromolecules, 2002, 35, 10131-10148.

81 T. Yaoita, T. Isaki, Y. Masubuchi, H. Watanabe, G. Ianniruberto and G. Marrucci, Macromolecules, 2011, 44, 9675-9682.

82 T. Yaoita, T. Isaki, Y. Masubuchi, H. Watanabe, G. Ianniruberto and G. Marrucci, Macromolecules, 2012, 45, 2773-2782.

83 P. S. Desai and R. G. Larson, J. Rheol., 2014, 58, 255-279.

84 R. K. Gupta, D. A. Nguyen and T. Sridhar, Phys. Fluids, 2000, 12, 1296-1318.

85 J. M. Wiest, Rheol. Acta, 1989, 28, 4-12.

86 O. Scrivener, C. Berner, R. Cressely, R. Hocquart, R. Sellin and N. S. Vlachos, J. Non-Newtonian Fluid Mech., 1979, 5, 475-495.

87 C. E. Sing and A. Alexander-Katz, Macromolecules, 2010, 43, 3532-3541.

88 R. G. Larson, J. Rheol., 2005, 49, 1-70.

89 A. Peterlin, J. Polym. Sci., Part B: Polym. Lett., 1966, 4, 287291.

90 S. Somani, E. S. G. Shaqfeh and J. R. Prakash, Macromolecules, 2010, 43, 10679-10691.

91 M. Shribak and R. Oldenbourg, Appl. Opt., 2003, 42, 30093017.

92 T. J. Ober, J. Soulages and G. H. McKinley, J. Rheol., 2011, 55, 1127-1159.

93 M. R. Mackley, Rheol. Acta, 2010, 49, 443-458.

94 L. Rayleigh, Proc. Lond. Math. Soc., 1878, s1-10, 4-13.

95 L. Rayleigh, Proc. R. Soc. London, 1879, 29, 71-97.

96 L. Rayleigh, Proc. R. Soc. London, 1882, 34, 130-145.

97 S. Middleman, Modeling Axisymmetric Flows: Dynamics of Films, Jets and Drops, Academic Press, San Diego, 1995.

98 F. W. Kroesser and S. Middleman, AIChE J., 1969, 15, 383386.

99 V. M. Entov, Arch. Mech., 1978, 30, 435-442.

100 S. Middleman, Chem. Eng. Sci., 1965, 20, 1037-1040.

101 D. W. Bousfield, R. Keunings, G. Marrucci and M. M. Denn, J. Non-Newtonian Fluid Mech., 1986, 21, 79-97.
102 G. Brenn, Z. B. Liu and F. Durst, Int. J. Multiphase Flow, 2000, 26, 1621-1644.

103 Z. H. Liu and Z. B. Liu, Int. J. Multiphase Flow, 2008, 34, 4260.

104 V. M. Entov and E. J. Hinch, J. Non-Newtonian Fluid Mech., 1997, 72, 31-54.

105 C. A. Schneider, W. S. Rasband and K. W. Eliceiri, Nat. Methods, 2012, 9, 671-675.

106 J. Odell, A. Keller and M. Miles, Polymer, 1985, 26, 12191226.

107 G. G. Fuller, Optical Rheometry of Complex Fluids, Oxford Univ. Press, 1995.

108 S. J. Haward, J. A. Odell, Z. Li and X.-F. Yuan, Rheol. Acta, 2010, 49, 781-788.

109 T. T. Perkins, D. E. Smith and S. Chu, Science, 1997, 276, 2016-2021.

110 D. E. Smith and S. Chu, Science, 1998, 281, 1335-1340.

111 F. Meyer, D. Lohmann and W. M. Kulicke, J. Rheol., 2009, 53, 799.

112 W. M. Kulicke, U. Reinhardt, G. G. Fuller and O. Arendt, Rheol. Acta, 1999, 38, 26-33.

113 M. R. Duxenneuner, P. Fischer, E. J. Windhab and J. J. Cooper-White, Biomacromolecules, 2008, 9, 2989-2996.

114 H. Tan, K. C. Tam, V. Tirtaatmadja, R. D. Jenkins and D. R. Bassett, J. Non-Newtonian Fluid Mech., 2000, 92, 167185.

115 F. Tanaka and S. J. Edwards, J. Non-Newtonian Fluid Mech., 1992, 43, 247-271.

116 C. A. Cathey and G. G. Fuller, J. Non-Newtonian Fluid Mech., 1988, 30, 303-316.

117 D. M. Jones, K. Walters and P. R. Williams, Rheol. Acta, 1987, 26, 20-30.

118 L. M. Quinzani, R. C. Armstrong and R. A. Brown, J. Rheol., 1995, 39, 1201-1228.

119 X. F. Li and M. M. Denn, J. Rheol., 2004, 48, 805-821.

120 J. K. Nielsen, H. K. Rasmussen, O. Hassager and G. H. McKinley, J. Rheol., 2006, 50, 453-476.

121 N. Sasaki, E. D. T. Atkins and W. S. Fulton, J. Appl. Polym. Sci., 1991, 42, 2975-2985.

122 S. Fujii, N. Sasaki and M. Nakata, Colloid Polym. Sci., 2003, 281, 823-831.

123 A. W. Chow and G. G. Fuller, Macromolecules, 1985, 18, 786793.

124 A. W. Chow, G. G. Fuller, D. G. Wallace and J. A. Madri, Macromolecules, 1985, 18, 793-804.

125 G. H. McKinley and A. Tripathi, J. Rheol., 2000, 44, 653-670.

126 E. Miller, C. Clasen and J. P. Rothstein, Rheol. Acta, 2009, 48, 625-639.

127 N. J. Kim, C. J. Pipe, K. H. Ahn, S. J. Lee and G. H. McKinley, J. Rheol., 2010, 22, 31-41.

128 M. Rubinstein and R. Colby, Polymer Physics, Oxford Univ. Press, New York, 2003.

129 C. Clasen, J. P. Plog, W. M. Kulicke, M. Owens, C. Macosko, L. E. Scriven, M. Verani and G. H. McKinley, J. Rheol., 2006, 50, 849-881.

130 J. Plog, W. Kulicke and C. Clasen, Appl. Rheol., 2005, 15, 2837. 
131 M. A. Fontelos and J. Li, J. Non-Newtonian Fluid Mech., 2004, 118, 1-16.

132 P. P. Bhat, O. A. Basaran and M. Pasquali, J. Non-Newtonian Fluid Mech., 2008, 150, 211-225.

133 R. Bird, P. Dotson and N. Johnson, J. Non-Newtonian Fluid Mech., 1980, 7, 213-235.

134 M. Renardy, J. Non-Newtonian Fluid Mech., 2002, 104, 65-74. 135 M. Renardy, Rheol. Rev., 2004, 2, 171-196.

136 D. C. Vadillo, S. D. Hoath, W.-K. Hsiao and M. R. Mackley, NIP \& Digital Fabrication Conference, 2011.

137 B. H. Zimm, J. Chem. Phys., 1956, 24, 269-278.

138 P. J. Flory and T. G. Fox, J. Am. Chem. Soc., 1951, 73, 19041908.

139 P. J. Flory, O. K. J. Spurr and D. K. Carpenter, J. Polym. Sci., 1958, 27, 231-240.
140 K. Saalwachter, W. Burchard, P. Klufers, G. Kettenbach, P. Mayer, D. Klemm and S. Dugarmaa, Macromolecules, 2000, 33, 4094-4107.

141 V. Tsvetkov, Russ. Chem. Rev., 1969, 38, 755-773.

142 K. Kamide, Cellulose and Cellulose Derivatives: Molecular Characterization and its Applications, Elsevier, Amsterdam, 2005.

143 D. F. James and T. Sridhar, J. Rheol., 1995, 39, 713-724.

144 C. N. Hoth, S. A. Choulis, P. Schilinsky and C. J. Brabec, Adv. Mater., 2007, 19, 3973-3978.

145 M. S. N. Oliveira and G. H. McKinley, Phys. Fluids, 2005, 17, 071704.

146 P. P. Bhat, S. Appathurai, M. T. Harris, M. Pasquali, G. H. McKinley and O. A. Basaran, Nat. Phys., 2010, 6, 625-631. 